\title{
Simulation of Dry Powder Inhalers: Combining Micro-Scale, Meso-Scale and Macro-Scale modeling
}

\author{
Berend van Wachem \\ Division of Thermofluids, Department of Mechanical Engineering \\ Imperial College London, Exhibition Road, London SW7 2AZ \\ B.van-Wachem@imperial.ac.uk \\ Kyrre Thalberg, Johan Remmelgas, Ingela Niklasson-Björn \\ Pharmaceutical development, AstraZeneca R\&D, 438183 Mölndal, Sweden
}

June 10, 2016

\begin{abstract}
The flow of carrier particles, coated with active drug particles, is studied in a prototype dry powder inhaler. A novel, multi-scale approach consisting of a discrete element model (DEM) to describe the particles coupled with a dynamic large eddy simulation (LES) model to describe the dynamic nature of the flow is applied. The model consists of three different scales: the micro-scale, the meso-scale and the macro-scale. At the micro-scale, the interactions of the small active drug particles with larger carrier particles, with the wall, with the air flow, and with each other is thoroughly studied using discrete element modelling and detailed computational fluid dynamics (CFD), i.e. resolving the flow structures around the particles. This has led to the development of coarse-grained models, describing the interaction of the small active drug particles at the larger scales.
\end{abstract}


At the meso-scale the larger carrier particles, and all of their interactions are modelled individually using DEM and CFD-LES. Collisions are modeled using a visco-elastic model to describe the local deformation at each point of particle-particle contact in conjunction with a model to account for cohesion.

At the macro-scale, simulations of a complete prototype inhaler are carried out. By combining the relevant information of each of the scales, simulations of the inhalation of one dose from a prototype inhaler using a patient relevant air flow profile show that fines leave the inhaler faster than the carrier particles. The results also show that collisions are not important for particle-particle momentum exchange initially but become more important as the particles accelerate. It is shown that for the studied prototype inhaler the total release efficiency of the fine particles is between $10 \%$ and $30 \%$, depending on the Hamaker constant, using typical settings for the properties of both particles. The results are also used to study regions of recirculation, where carrier particles can become trapped, and regions where fines adhere to the wall of the device.

Keywords: Dry powder inhalers (DPI), Computational Fluid Dynamics (CFD), Large eddy simulation (LES), Discrete element models (DEM) 


\section{List of Figures}

1 A sketch of the torque arising from: (a) rolling and (b) torsion. . . . . . . . . . . . . . 42

2 A sketch of the $\mathrm{DMT}^{1}$ adhesion model: a contact point which results in an elastic contact force, and the adhesion force outside of the direct contact point. . . . . . . . . . 43

3 The result of a carrier particle coated with the 2,000 fine particles. . . . . . . . . . . . . 44

4 The distribution of clusters of fines on the carrier particle surface. . . . . . . . . . . 45

5 The four "micro-model" mechanisms studied in the present work: 1. Particle-particle collision, 2. Particle-wall collision, 3. Detachment of particles by fluid flow, and 4 . re-attachment of fines. . . . . . . . . . . . . . . . . . . . 46

6 The percentage of small particles removed as a function for normal impact velocity for various $H a$ constants. The dashed lines represent the results predicted by Equation 40 .

7 The simulation results (symbols) and the empirical fit for the fraction of fine particles that are removed from the carrier particle for $H a=1.0 \times 10^{-19} \mathrm{~J}$ as a function of normal impact velocity with a wall. . . . . . . . . . . . . . . . . . .

8 The simulation results (symbols) and the fit for the fraction of fine particles that are removed from the carrier particle and deposited onto the wall for $H a=1.0 \times 10^{-19} \mathrm{~J}$ as a function of normal impact velocity with a wall. . . . . . . . . . . . . . .

9 The simulation results (symbols) and the fit for the number of fine particles that are removed from the wall when it is hit by a carrier particle for $H a=1.0 \times 10^{-19} \mathrm{~J}$ as a function of the relative concentration of fines on the wall. . . . . . . . . . . . 50

10 A sketch of the geometry of the inhaler and the initial placement of the particles. . . . . 51

11 The geometry and the surface mesh of the inhaler geometry. . . . . . . . . . . . . 52

12 The flow rate, in $l / \min$, through the inhaler as a function of time. . . . . . . . . . 53

13 A sequence of two-dimensional cross sectional visualisations of the inhaler, showing the air velocity and the location of the individual carrier particles (coloured red, $d_{p}=70 \mu m$ ) for different times. 
14 The number of carrier particles (red, left Y-axis) and the number of fine particles (blue, right $\mathrm{Y}$-axis) exiting the inhaler as a function of time. . . . . . . . . . . 55

15 The cumulative number of fines that are released from the carrier particle as they exit the inhaler as a function of time, for different Hamaker values. . . . . . . . . . . . . 56

16 The cumulative number of fines that exit the inhaler, which are still attached to a carrier

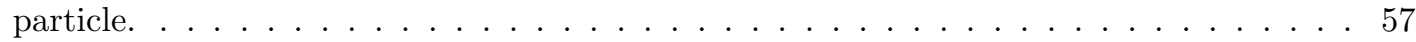

17 The cumulative number of fines that exit the inhaler, either attached to a carrier particle (dashed lines) or released (solid lines) for the case including the blow-off mechanism compared to excluding this mechanism. The results shown are for withes with Hamaker constant $H a=5.0 \cdot 10^{-19} J \ldots \ldots \ldots \ldots \ldots \ldots$. . . . . . . . . . . . . . 58

18 The location and relative concentration of the fine particles which remain adhered to the wall after all carrier particles have left the inhaler. The results shown are for the fine particles with $H a=1.0 \cdot 10^{-19} \mathrm{~J} \ldots \ldots \ldots \ldots \ldots \ldots$. . . . . . . . . 59

19 The location and relative contribution to detachment of fine particle release due to particles colliding with the wall. The results shown are for the fine particles with $H a=1.0 \cdot 10^{-19} J \ldots \ldots \ldots \ldots \ldots \ldots \ldots$

20 The location and relative contribution to detachment of fine particle release due to particle-particle collisions. The top image, side view, shows the cross-section through the middle of the inhaler. The results shown are for the fine particles with $H a=1.0 \cdot 10^{-19} \mathrm{~J}$. 61

21 The location and relative net contribution to the detachment of fine particles for all the micro-model mechanisms considered in this paper. The top image, side view, shows the cross-section through the middle of the inhaler. The results shows are for the fine particles with $H a=1.0 \cdot 10^{-19} J \ldots \ldots \ldots \ldots \ldots$ 


\section{Introduction}

Inhalation of medicinal drugs using dry powder inhalers (DPIs) is a well established technology for drug delivery to the lungs. The first products entered the market more than 40 years ago and sales of products based on DPIs amount to approximately 10 billion USD in 2015, growing at an average five year annual growth rate of $12.5 \%$. DPIs consist of a formulation in the form of a dry powder and an inhalation device. They are attractive because the drug substances are kept in a dry form, which is advantageous from the point of view of the stability, because they are easy to use as dose release is automatically coupled with the patient's inhalation, and because they do not rely on using a propellant. $^{2}$

From a purely theoretical point of view it would be advantageous to employ a formulation consisting solely of particles with a diameter smaller than approximately $5 \mu m$ since particles with a diameter greater than this typically are deposited in the upper airways and do not reach the lungs. In practice, however, the high specific surface area of such particles usually implies that cohesive forces become dominant. Thus, formulations based upon small particles tend to flow poorly and are thus very difficult to fill into the device with any kind of accuracy or precision.

Formulations for inhalation are therefore often developed by incorporating larger carrier particles. During mixing, the drug substance adheres to the carrier particles, and they are re-dispersed into the air stream during the inhalation process. ${ }^{2}$ The device thus plays a very important role in dispersing the powder into small particles and the performance of the device, with regards to powder dispersion and retention, is a major concern during device development. Of course, the device and the formulation cannot be developed independently of one another and it is sometimes found that a device that works well for one type formulation does not work for another.

Extensive research and development of DPIs, have demonstrated the large complexity of these sys- 
tems. $^{3,4}$ In fact, both the formulation itself and the interaction between formulation and the device during inhalation are poorly understood. In particular, there is presently no theory that can be employed to reliably predict the fine particle fraction of a formulation from a DPI. Numerous articles in the literature have discussed and compared the performance of different devices ${ }^{5-8}$ and even more work has been directed to comparing different formulations or drug substance, ${ }^{9-18}$ but no approach that can provide a quantitative link between the properties of the particles to the performance of the device has yet been proposed. This field is also still very much in development, with novel opportunities, such as porous particles and engineered composite particles applied. ${ }^{19,20}$

While models exist that can be used to describe the interaction between a small number of particles, these models cannot be directly applied to describe DPIs, simply because the number of particles is too large. For example, $1 \mathrm{mg}$ of drug substance in the form of spheres with a diameter of $1 \mu \mathrm{m}$ corresponds to $10^{9}$ particles, which is far beyond what is practical for computer simulations. Since the mass of a particle scales $d_{p}^{3}$, it is readily realized that $1 \mathrm{mg}$ of carrier particles with a diameter of $100 \mu \mathrm{m}$ corresponds to only $10^{3}$ particles. DPIs thus offer a unique opportunity to capitalize on recent progress in computer and computational science since it is now possible, though still difficult, to simulate the fate of every carrier particle using computational fluid dynamics (CFD) and discrete element models (DEM).

Computational fluid dynamics (CFD) has been applied to resolve the air flow pattern in a number of inhalation devices and to study the motion of particles. ${ }^{21-23}$ While simulations excluding the effect of particle-particle interactions have now become mainstream, the inclusion of a dry powder for which particle-particle interactions cannot be neglected makes the situation much more complicated. Even more so if the behaviour of the fines is taken into account as well. Turbulence modelling becomes particularly complicated for DPIs since the characteristic length scale of the devices usually implies that the Reynolds number of the flow is, at the highest flow-rate, on the order of $10^{4}$, and thus can be 
expected to undergo a transition from laminar to turbulent during the inhalation event when the flow rate increases from 0 to a peak inspiration rate of $70 \mathrm{l} / \mathrm{min}$. This latter effect is not considered at all in the studies that rely on Reynolds-averaged Navier-Stokes (RANS) models to model turbulence. ${ }^{22,23}$

In this work, we therefore develop a fundamental approach to simulate dose emptying and dispersion in a model dry powder inhaler that explicitly takes into account the interaction between carrier particles, that may be cohesive or adhesive, and their interaction with a possibly turbulent velocity field. The CFD model thus employed is based upon a dynamic large eddy simulation (LES) model, ${ }^{24,25}$ in which the energetic flow scales are fully resolved, and the smallest scales are modelled using a subgrid scale (SGS) model. Using this CFD framework, the entrainment and subsequent journey of each individual carrier particle through the inhaler is resolved. The behavior of every single carrier particle is determined, and both the effect of the carrier particles on the airflow and the interaction between carrier particles are taken into account. In addition, the detachment and possible re-attachment of small drug particles from the carrier particles is dynamically determined during dose emptying. This approach thus provides a quantitative link between the properties of the particles and the performance of the device. Using this model, it is hoped that key measures of pharmaceutical performance, such as the drug fine particle fraction and the amount retained in the device, may be predicted.

In order to develop a model that can provide a quantitative link between the properties of the particles and the performance of the device, a three stage procedure is adopted:

1. Simulations of the fine particles which are attached to one or a small number of carrier particles are used to study the dynamics of the detachment and re-attachment from carrier particles. This study will be referred to as "micro-models", and include attachment of fine particles onto the carrier, detachment of fine particles from the carrier particles due to air flow, carrier - carrier particle collisions, or carrier particle - wall collisions and re-attachment of released fine particles back onto carrier particles. 
2. Carrier particle entrainment and emptying of the inhaler is simulated using a LES-CFD-DEM approach. Each individual carrier particle is tracked throughout the domain, and all collisions with the walls and with other carrier particles are resolved and tracked.

3. The results from the micro-models are combined with the carrier particles emptying at every single time-step of the simulation. In this way, the state of the fine particles (free or attached) as well as the travel of every single carrier particle is followed throughout the simulation.

This three-staged framework is here applied to a simple prototype inhaler using a patient relevant inhalation profile. Key output measures obtained are: airflow development and the movement of carrier particles through the inhaler, emptying profiles for fine and carrier particles, the net fraction of fine particles released, i.e. the fine particle fraction, and the amount and location of particles retained in the inhaler.

This paper is organised in six sections. The first section describes how the equation of motion for each particle is solved, the DEM algorithm, and describes how the fluid-phase is solved and how the fluid sub-grid scales are modelled. The second section describes the development of the "micro-scale" models and presents the subsequent results. Subsequently, the simulation domain and set-up are described. The final sections present the numerical results and several aspects of the findings and summarise the main conclusions of this work.

\section{Particle and Fluid Modelling}

\section{Equations of Motion of the Particles}

Newton's $2^{\text {nd }}$ law for a particle in a gas can be written as

$$
m_{p} \frac{D \boldsymbol{v}_{p}}{D t}=\beta \frac{V_{p}}{\alpha_{p}}\left(\boldsymbol{v}_{f @ p}-\boldsymbol{v}_{p}\right)+m_{p} \boldsymbol{g}-V_{p} \nabla P+\boldsymbol{F}_{p w}+\boldsymbol{F}_{p p}
$$


where the term on the left hand side represents the acceleration of the particle, and the terms on the right hand side are the drag force, the gravity force, the buoyancy force, the forces arising from particlewall interactions $\left(\boldsymbol{F}_{p w}\right)$, and the forces arising from particle-particle interactions $\left(\boldsymbol{F}_{p p}\right)$, respectively. The added mass force, lift forces and history forces are neglected because the density ratio between the air and the particles is very large. ${ }^{26}$ The mass of the particle is $m_{p}, V_{p}$ is the volume of a particle, $\boldsymbol{v}_{f @ p}$ is the undisturbed fluid velocity at the particle, $\boldsymbol{v}_{p}$ is the particle translational velocity, $\nabla P$ is the local fluid pressure gradient, and $\beta$ is the reciprocal drag time, ${ }^{27}$ where the reciprocal of the Eulerian fluid-particle timescale is given by

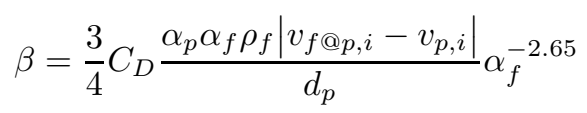

and $C_{D}$ represents the coefficient of drag for an individual particle and $\alpha_{f}$ represents the fluid volume fraction. The coefficient of drag, $C_{D}$, is defined $\operatorname{as}^{28}$

$$
C_{D}=\left\{\begin{array}{cc}
\frac{24\left[1+0.15\left(\left(1-\alpha_{p}\right) R e_{p}\right)^{0.687}\right]}{R e_{p}\left(1-\alpha_{p}\right)} & , \text { if }\left(1-\alpha_{p}\right) R e_{p}<1000 \\
0.44 & , \text { if }\left(1-\alpha_{p}\right) R e_{p} \geq 1000
\end{array}\right.
$$

The equation for rotational acceleration for each particle is given by

$$
I \frac{d \boldsymbol{\omega}}{d t}=\boldsymbol{T}_{p}
$$

where $I$ is the moment of inertia of the particle, $\boldsymbol{\omega}$ is the rotational velocity of the particle, and $\boldsymbol{T}_{p}$ is the sum of external torques, arising from particle-wall and particle-particle interactions. 


\section{Integration of the equations of motion}

The Verlet scheme is used to integrate the equation of motion for a particle in order to obtain the position and velocity vectors after a collision. ${ }^{29}$ The position vector is updated by

$$
\boldsymbol{x}_{p}(t+\Delta t)=2 \boldsymbol{x}_{p}(t)-\boldsymbol{x}_{p}(t-\Delta t)+\Delta t^{2} \frac{\boldsymbol{F}_{p}(t)}{m_{p}}+O\left(\Delta t^{4}\right)
$$

where $\boldsymbol{F}_{p}(t)=\boldsymbol{F}_{n}(t)+\boldsymbol{F}_{t}(t)$ and $\Delta t$ is the applied numerical time-step. The error of equation (5) is of $O\left(\Delta t^{4}\right)$

The velocity vector of the particles is obtained via

$$
\boldsymbol{v}_{p}(t)=\frac{\boldsymbol{x}_{p}(t+\Delta t)-\boldsymbol{x}_{p}(t-\Delta t)}{2 \Delta t}+O\left(\Delta t^{2}\right)
$$

A similar integration scheme is adopted to update the rotational acceleration, as given by Equation 4 . The applied time-step, $\Delta t$, has to be sufficiently small that the linearisation applied above is accurate. In this work, any particle-particle interaction is resolved using at least 40 integration time steps.

\section{Distinct Element Modelling}

The particle-particle and particle-wall interactions are solved using a soft-sphere distinct element model. The soft sphere model was first proposed by Cundall and co-workers. ${ }^{30}$ In this framework, particles are allowed to overlap slightly at the points of contact, where the amount of overlap is directly proportional to the amount of deformation at the contact point of the two particles. The soft-sphere model allows for multiple as well as enduring contacts for each particle; the net contact force at any point in time is given by the sum of all the pair-wise interactions occurring at that time. Each interaction consists of a force in the normal direction and a force in the tangential direction of the unit contact vector. The contact vector is given by the vector perpendicular to the contact plane. Mathematically, the contact model can be interpreted as a non-linear spring-dashpot-slider framework to describe the 
particle behaviour during a collision. The soft sphere model takes into account the actual deformation of the particles and determines the resulting normal and tangential forces using the physical parameters of the particles. These forces are determined by the actual material type and properties and can be, for instance, elastic, plastic, or visco-elastic. ${ }^{31}$ In the current work the forces are determined using visco-elastic theory.

\section{Contact forces}

The normal and tangential contact forces are given by the contact model with appropriate particle properties. From Hertzian contact theory combined with visco-elastic damping the normal and tangential contact forces are ${ }^{32}$

$$
\begin{gathered}
\boldsymbol{F}_{n}=-k_{n} \delta^{\frac{3}{2}} \boldsymbol{n}-\eta_{n} \boldsymbol{q}_{n} \\
\boldsymbol{F}_{t}= \begin{cases}-k_{t} \boldsymbol{\delta}_{t}-\eta_{t} \boldsymbol{q}_{t} & \text { if sticking }\left(\left|\boldsymbol{F}_{t}\right|<\mu\left|\boldsymbol{F}_{n}\right|\right) \\
-\mu\left|\boldsymbol{F}_{n}\right| \frac{\boldsymbol{q}_{t}}{\left|\boldsymbol{q}_{t}\right|} & \text { if sliding }\left(\boldsymbol{F}_{t}|\geq \mu| \boldsymbol{F}_{n} \mid\right)\end{cases}
\end{gathered}
$$

The subscripts $n$ and $t$ represent the normal and tangential components respectively and $\delta$ is the displacement, or overlap, during the collision of two particles, representing the actual deformation occurring in the contact point. The vector $\boldsymbol{n}$ represents the unit collision normal, a unit vector perpendicular towards the contact plane, and the vector $\boldsymbol{q}$ represents the relative velocity between the particles in the contact plane. The vector $\boldsymbol{\delta}_{t}$ represents the cumulative tangential displacement determined by integrating the incremental tangential displacements mapped onto the current collision plane. Two parameters for the normal and three parameters for the tangential directions are required by the soft sphere model: stiffness $\left(k_{n}\right.$ and $\left.k_{t}\right)$, damping $\left(\eta_{n}\right.$ and $\left.\eta_{t}\right)$ and friction $(\mu)$ coefficients. The damping coefficients are related to the appropriate material properties of the particle:

$$
\eta_{n}=\alpha \sqrt{M k_{n}} \delta^{\frac{1}{4}}
$$




$$
\eta_{t}=\alpha \sqrt{M k_{t}}\left|\boldsymbol{\delta}_{t}\right|^{\frac{1}{4}}
$$

where $M=\frac{m_{p_{1}} m_{p_{2}}}{m_{p_{1}}+m_{p_{2}}}$; where $m_{p}$ represents the mass of each of the particles. Note that for wall collisions the same equation holds, as a wall can be considered as a particle with infinite mass and radius. The relationship between the coefficient of restitution $e$ and the parameter $\alpha$ is defined as dampening, ${ }^{32}$ and will depend upon the impact velocity, as is expected for a visco-elastic material. The parameter $\alpha$ is non-linearly related to $e$, which makes it independent of mass, Young's modulus, Poisson ratio and the coefficient of friction. The stiffness coefficients for the normal and tangential directions are given, based on elastic deformation, by

$$
\begin{gathered}
k_{n}=\frac{4}{3}\left(\frac{1-\sigma_{p_{1}}^{2}}{E_{p_{1}}}+\frac{1-\sigma_{p_{2}}^{2}}{E_{p_{2}}}\right)^{-1}\left(R^{*}\right)^{\frac{1}{2}} \\
k_{t}=8\left(\frac{2-\sigma_{p_{1}}}{G_{p_{1}}}+\frac{2-\sigma_{p_{2}}}{G_{p_{2}}}\right)^{-1}\left(R^{*}\right)^{\frac{1}{2}} \delta^{\frac{1}{2}}
\end{gathered}
$$

where the reduced radius is given by

$$
R^{*}=\frac{r_{p_{1}} r_{p_{2}}}{r_{p_{1}}+r_{p_{2}}}
$$

and where $r_{p}$ is the radius of the particle, $\sigma$ Poisson's ratio, $E$ the Young's modulus, $G$ the shear modulus (given by $G=\frac{E}{2(1+\sigma)}$ ) and $\delta_{n}$ the magnitude of the deformation in the normal direction. When a particle collides with a wall, the wall is considered as a particle with infinite radius and mass, hence, $R^{*}=r_{p_{1}}$. The friction coefficient, $\mu$ is a material parameter which is often taken as a constant.

\section{Rolling and torsion resistance}

Although friction is included in the above framework, there is no mechanism to counteract the rolling of a particle. When a particle rolls on a surface, the relative tangential velocity is zero and the resultant frictional force is zero. Although this is realistic for perfect spheres with minimal deformation, in practice a rolling resistance is present due to the non-ideal nature of the particles. To account for the surface roughness and non-spherical nature of real materials, a rolling friction or rolling resistance 
and a torsion resistance has been included. These resistances are illustrated in Figure 1. The simplest models approximate these resistances as a torque that opposes the rotation and depends linearly on the ongoing rotation in a dissipative faction, ${ }^{33}$ thus

$$
\tau_{d}=\mu_{d}\left|\boldsymbol{F}_{n}\right| \frac{\boldsymbol{\omega}}{|\boldsymbol{\omega}|}
$$

where $\mu_{d}$ is the coefficient of rolling friction with units of length, $\boldsymbol{\omega}$ is the relative angular velocity of the particle, and $\boldsymbol{F}_{n}$ is the normal force acting in the contact area. However, such a simple model is not very realistic because it does not account for the physics occurring at the contact area.

The sources of a rolling resistance should be considered by partial sticking and by micro-roughness of contact surfaces and by contact deformations. The rolling moment for elastic-frictional behaviour originates from the non-linear elastic contribution for partial sticking within the contact plane. This thus depends on the load dependent force for unloading at any positive unload point and rolling in the reverse direction. ${ }^{34}$

A rolling particle experiences a torque which opposes its rolling motion. ${ }^{34,35}$ The opposing torque is comprised of two components: $\tau_{\theta}$, which relates to the total angle rolled, and a dissipative term, $\tau_{d}$, which is proportional to the angular velocity of rolling (see Equation 14),

$$
\tau_{r}=\tau_{\theta}+\tau_{d}
$$

The first component, $\tau_{\theta}$, bears a strong resemblance to the Mindlin-Deresiewicz model of friction. ${ }^{36}$ For a two dimensional system, in each time-step the angle rolled between two particles is determined by their respective angular velocities:

$$
\Delta \theta_{r}=\Delta t\left(\omega_{1}-\omega_{2}\right)
$$


where $\omega_{i}$ is the angular velocity of particle $i$, and $\Delta t$ is the timestep of integration. The total angle rolled can then be calculated incrementally, by summing the contribution in each timestep. Thus for time-step $n$

$$
\theta_{r}^{n}=\theta_{r}^{n-1}+\Delta \theta_{r}^{n}
$$

Up until a critical angle, $\theta_{0}$, the torque is proportional to $\theta_{r}$. This critical angle is an empirical parameter of the model. Beyond this angle it takes a constant value of $\tau_{0}=\mu_{\theta} R^{*} F_{n}$, where $\mu_{\theta}$ is the coefficient of rolling resistance, which is an empirical parameter, $R^{*}$ is the reduced radius, and $F_{n}$ is the normal force acting at the collision. To ensure continuity of $\theta_{r}$, in the region where $\theta_{r}<\theta_{0}$, the constant of proportionality is defined as $k_{r}=\frac{\tau_{0}}{\theta_{0}}$. The torque is thus related to $\theta_{r}$ by the relationship

$$
\tau_{\theta}= \begin{cases}-k_{r} \theta_{r} & \text { if }\left|\theta_{r}\right|<\theta_{0}, \\ -\tau_{0} \frac{\theta_{r}}{\left|\theta_{r}\right|} & \text { otherwise. }\end{cases}
$$

If the particle starts to roll back in the opposite direction, i.e. if $\theta_{r} \Delta \theta<0$, the torque should fall linearly back to 0 such that the particle may roll a distance before coming to a halt. It is therefore useful to limit the magnitude of $\theta_{r}$ to $\theta_{0}$, resulting in the required behaviour for $\tau_{\theta}$ with no further modification. With this modification equation 17 becomes

$$
\theta_{r}^{n}=\min \left(\theta_{r}^{n-1}+\Delta \theta_{r}^{n}, \theta_{0}\right)
$$

Torsion Resistance When two elastic spheres in contact under constant normal force, $F_{n}$, are subject to a torsion about the axis of their common normal, relative motion of the spheres is resisted by frictional interactions, see Figure 1(b). In a similar manner to the tangential loading of elastic spheres, the torsion leads to a region of slip at the interface. The slipped area starts at the edge of the contact and creeps inwards as the relative slip angle, $\beta$, between the surfaces increases. This behaviour can be described mathematically. ${ }^{37}$ 
The angle of twist for an applied torque, $\tau_{z}$, is given by

$$
\beta=\frac{3}{16} \frac{\tau_{z}}{G^{*} a^{3}}
$$

where $G^{*}=\frac{G_{1} G_{2}}{G_{1}+G_{2}}, G_{i}$ is the shear modulus of particle $i$, and $a$ is the contact radius. The contact radius $a$ can be calculated using the Hertz theory of elastic contact:

$$
a=\left(\frac{3 F_{n} R^{*}}{4 E^{*}}\right)^{\frac{1}{3}}
$$

where $R^{*}$ is the effective radius at the contact and $E^{*}$ is the reduced elastic modulus, $E^{*}=\frac{E_{1} E_{2}}{E_{1}\left(1+\nu_{2}^{2}\right)+E_{2}\left(1+\nu_{1}^{2}\right)}$. $\nu_{i}$ is the Poisson ratio of particle $i$. When the entire contact has slipped, further twisting motion is resisted by a constant torque of

$$
\tau_{z}^{\max }=\frac{3 \pi \mu F_{n} a}{16}
$$

The angle at which this occurs can be calculated using equation 20 . In the region between $\beta=0$ and $\beta=\beta^{\max }$ the torque acting between the spheres increases linearly from 0 to $\tau_{z}^{\max }$. When the angle $\beta^{\max }$ is reached, the torque remains constant. The gradient of this increase, $k_{t}$, is given by:

$$
\begin{aligned}
k_{t} & =\frac{\tau_{z}^{\max }}{\beta^{\max }} \\
& =\frac{16}{3} G^{*} a^{3},
\end{aligned}
$$

For each collision in a DEM simulation it is necessary to keep track of the total angle of relative rotation about the collision normal. A torque opposing this motion increases linearly to $\tau_{z}^{\max }$ as while this angle is less than $\beta^{\text {max }}$. If the direction of relative spin motion is reversed, the torque should fall linearly to zero, and continue to a maximum negative value of $-\beta^{\text {max }}$. It is therefore not useful to continue to increase $\beta$ beyond $\beta^{\max }$. 


\section{Adhesion}

Small micro-particles are attached to a larger carrier particle by means of adhesion of the type of van der Waals forces. ${ }^{38}$ An important concept of two particles which are cohesively attached together, is the force required to remove the particles from each other, i.e. the total adhesion force. This is defined as the pull-off force. ${ }^{38}$ Although other mechanisms of adhesion exist, such as electrostatic or liquid bridges, this paper only considers van der Waals type of adhesion.

One of the models to determine the magnitude of the adhesion force is the DMT model, ${ }^{1}$ which is adopted in this work. In the DMT model, the molecular forces causing the cohesion are calculated based on the assumption that the deformed profiles of the bodies are given by the Hertz theory. The adhesion energy in the area of contact is equal to the non contacting parts on the surface, see Figure 2. In this approximation the deformation of the spheres outside the area of contact due to the attractive van der Waals forces is neglected. Figure 2 shows the pull off force when a sphere collides with a flat surface. The pull-off force is given by

$$
F_{0}=4 \pi a \gamma
$$

where $\gamma$ is the energy at the surface and $a$ is the net radius of the contact area of the two particles. This radius can be determined from the overlap of the two particles. If the cohesion is caused by a Van der Waals force, the surface energy of a sphere in contact with a plane is given by

$$
\gamma=\frac{H a}{24 \pi \delta_{0}^{2}}
$$

where $H a$ is the Hamaker coefficient and $\delta_{0}$ is the molecular equilibrium distance of the molecules in the contact region. When two spherical particles are in cohesive contact, the cohesive force, $F_{c}$, in the 
direction of the contact normal, by the DMT model is determined as

$$
F_{c}= \begin{cases}F_{0}\left[1-\frac{3 \sqrt{2}}{4}\left(\frac{\delta}{\delta_{0}}\right)^{\frac{1}{2}}+\frac{\delta}{4 \delta_{0}}\right] & \text { if } \delta<\delta_{0} \\ \frac{F_{0}}{2} & \text { if } \delta>\delta_{0}\end{cases}
$$

The above cohesion force is applied to particles already in contact and is added to the forces resulting from the visco-elastic models of the contact.

\section{Fluid Phase Modelling}

There are various frameworks in which the continuous phase for gas-solid flows can be predicted, i.e. Direct Numerical Simulation (DNS), Large Eddy Simulation (LES) and the Reynolds Averaged Navier-Stokes (RANS) method. DNS methods offer high accuracy in resolving all scales without adhoc modelling at the expense of enormous computational time. Currently, DNS can only solve flows of relatively low Reynolds $(R e)$ numbers, which are outside of most engineering and industrial interests. On the other hand, in RANS all of the scales are modelled using turbulence models. Although this is a very affordable approach, the prediction capabilities of this framework are limited, especially for intermediate Reynolds number flows in complex geometries, such as an inhaler. Between DNS and RANS lies LES. Although the computational effort for LES is still very high, it is considerably lower than for DNS and it has therefore become very fashionable for analysing flows in academia and it is also an emerging tool in industry.

An LES model solves the Navier-Stokes equations up to a particular length-scale due to the application of a filter. Length-scales smaller than the cut-off filter width $(\Delta)$ are modelled with a so-called sub-grid scale (SGS) model. The cut-off width is an indication of the smallest size eddies that are retained in the computations and eddies smaller than $\Delta$, are filtered out. Due to the filtering of the Navier-Stokes equations, models are required to provide closure for the SGS stresses, which account 
for the effect of the unresolved scales on the convective momentum transport. More background information on the the filtering procedure for LES can be found in Sagaut. ${ }^{39}$ The equations arising from filtering are very similar to the Navier-Stokes equations, except for the addition of one term, which describes the behaviour of the sub-grid scale stresses, namely $\tau_{i j}^{a}$

$$
\tau_{i j}^{a}=\rho_{f}\left(\widetilde{v_{f, i} v_{f, j}}-\widetilde{v}_{f, i} \widetilde{v}_{f, j}\right)
$$

where the notation $\widetilde{v}_{f, j}$ represents the filtered value of the local and instantaneous velocity $v_{f, j}$. Thus, the filtered momentum equations for the fluid phase are

$$
\begin{aligned}
\frac{\partial\left(\alpha_{f} \rho_{f} \widetilde{v}_{f, j}\right)}{\partial t}+\frac{\partial\left(\alpha_{f} \rho_{f} \widetilde{v}_{f, j} \widetilde{v}_{f, i}\right)}{\partial x_{i}}= & -\alpha_{f} \frac{\partial \widetilde{p}}{\partial x_{j}}+\frac{\partial\left(\alpha_{f} \widetilde{\tau}_{i j}\right)}{\partial x_{i}}-\frac{\partial\left(\alpha_{f} \tau_{i j}^{a}\right)}{\partial x_{i}} \\
& +\sum_{p=1}^{\text {phases } \neq f} \beta_{(f, p)}\left[\widetilde{v}_{f @ p, j}-v_{p, j}\right]
\end{aligned}
$$

where $\alpha_{f}$ is the fluid volume fraction, $\rho_{f}$ is the fluid density and $\widetilde{v}_{f, i}$ is the filtered fluid velocity. The last term on the right hand side of Equation 29 are source terms; $\sum_{p=1}^{\text {phases } \neq f} \beta_{(f, p)}\left[\widetilde{v}_{f @ p, j}-v_{p, j}\right]$ is the inter-phase momentum exchange between the two phases respectively; the subscript $f @ p$ indicates the undisturbed fluid at the location of the particle.

Dynamic Germano-Lilly model The sub-grid scale (SGS) stresses are given as

$$
\tau_{i j}^{a}=-2 \mu_{S G S} \widetilde{S}_{i j}+\frac{1}{3} \tau_{l l} \delta_{i j}
$$

where $\widetilde{S}_{i j}=\frac{1}{2}\left[\frac{\partial \widetilde{v}_{f, i}}{\partial x_{j}}+\frac{\partial \widetilde{v}_{f, j}}{\partial x_{i}}\right]$. and $\mu_{S G S}$ is the sub-grid scale viscosity. By analogy to Prandtl's mixing-length hypothesis, ${ }^{40} \mu_{S G S}$ can be estimated as

$$
\mu_{S G S}=\rho_{f}\left(C_{S G S} \Delta\right)^{2} \sqrt{2 \widetilde{S}_{i j} \widetilde{S}_{i j}}
$$


where $C_{S G S}$ is the Smagorinsky constant and $\Delta$ is the LES filter width.

Although the Smagorinsky model has been used successfully in many turbulent flows, in complex wall-bounded turbulent flows, such as in a DPI, it lacks the required universality character needed to model turbulent flows. ${ }^{24}$ In addition, it does not take into account the energy back-scatter (i.e. the energy flow from small scales to large scales) which in some flows is important. ${ }^{24}$ Hence, Germano et $a l^{24}$ propose an algorithm which makes the Smagorinsky constant a function of space and time by assuming similarity of the filtered structures and applying a second coarser filter (or test filter) to the filtered momentum equation.

In the dynamic Germano-Lilly model, a new filtered shear stress tensor is formed, $T_{i j}$

$$
T_{i j}=\rho_{f}\left(\widehat{\widehat{v_{f, i} v_{f, j}}}-\widehat{\widetilde{v}}_{f, i} \widehat{\widetilde{v}}_{f, j}\right)
$$

Equation 32 is modelled using the same way as the sub-grid scale stresses of the Smagorinsky model, i.e.

$$
T_{i j}=-2 \widehat{\mu_{S G S}} \widehat{\widetilde{S}}_{i j}+\frac{1}{3} T_{l l} \delta_{i j}
$$

The resolved turbulent stresses $L_{i j}$ are defined as

$$
\begin{aligned}
L_{i j} & =T_{i j}-\widehat{\tau_{i j}^{a}} \\
& =\rho_{f}\left(\widehat{\widetilde{v}_{f, i} \widetilde{v}_{f, j}}-\widehat{\widetilde{v}}_{f, i} \widehat{\widetilde{v}}_{f, j}\right)
\end{aligned}
$$

Hence by subtracting equation 33 from equation $30, L_{i j}$ becomes

$$
L_{i j}=-2 \widehat{\mu_{S G S}} \widehat{\widetilde{S}}_{i j}+2 \mu_{S G S} \widetilde{S}_{i j}+\frac{1}{3} L_{l l} \delta_{i j}
$$


where

$$
\widehat{\mu_{S G S}}=\rho_{f}\left(\widehat{C_{S G S} \Delta}\right)^{2} \sqrt{2 \widehat{\widetilde{S}}_{i j} \widehat{\widetilde{S}}_{i j}}
$$

by assuming that $\left(\widehat{C_{S G S} \Delta}\right)^{2}=C_{S G S}^{2} \widehat{\Delta^{2}}, L_{i j}$ simplifies to

$$
L_{i j}=-2 \rho_{f} C_{S G S}^{2} M_{i j}+\frac{1}{3} L_{l l} \delta_{i j}
$$

where

$$
M_{i j}=\widehat{\Delta^{2}} \sqrt{2 \widehat{\widetilde{S}}_{i j} \widehat{\widetilde{S}}_{i j}} \widehat{\widetilde{S}}_{i j}-\Delta^{2} \sqrt{2 \widetilde{S}_{i j} \widetilde{S}_{i j}} \widetilde{S}_{i j}
$$

Solving equation 37 results in six independent $C_{S G S}$, hence a least-squares method is used to obtain one value ${ }^{41}$ (with small deviation from the other six). Thus, $C_{S G S}$ at each point and time is

$$
C_{S G S}=\frac{1}{2} \frac{L_{i j} M_{i j}}{M_{i j} M_{i j}}
$$

The above equation for the sub-grid scale constant can be directly used to determine the sub-grid scale viscosity, as given by equation 31. By calculating the SGS stresses, Equation 30, the momentum equations, Equations 29 are then solved in a fully-coupled manner. More details of this procedure are discussed in. ${ }^{25}$

\section{Micro-scale models}

The development of the micro-models starts by the creation of a carrier particle coated with fines, similar to what has been previously reported..$^{22,23}$ The carrier particle has a diameter of $d_{c}=70 \mu m$ and the fines have a diameter of $d_{f}=2.0 \mu m$ The properties of the carrier particle and the fine particles are taken to be those of lactose and are shown in Table 1.

Initially, 2,000 fine particles are deposited onto a carrier particle. It was found that a satisfactory way to add the fines to a carrier particle is to dynamically move the fines towards the carrier particle, 
while the carrier particle rotates. In this way, a large number of samples have been made, varying the Hamaker constant, Ha. The range of applied Hamaker constants is representative for lactose.42 It is assumed that the Hamaker constant for the carrier particle with the fine particle is the same as for the fine particle with another fine particle. The samples made this way are all quite similar in appearance, although there is a dependency on the rotation velocity of the carrier particle. The result for the particle rotating with $\omega=5 \cdot 10^{3} \mathrm{rad} / \mathrm{s}$ and the small particles approaching with a velocity of $U_{f}=0.1 \mathrm{~ms}^{-1}$ is shown in Figure 3. Although there is a tendency to form clusters on the carrier particle surface, this procedure of depositing fine particles on the carrier results in a fairly homogeneous distribution of fines on the carrier particle that is representative of a typical formulation. The resulting distribution of fines is shown in Figure 4. This coated carrier particle is used as the standard for all further micro-scale simulations. Four mechanisms for detachment and re-attachment of fines are researched using micro-scale models, by using the coated carrier particle under 4 different situations:

1. Detachment of fines by particle-particle collisions.

2. Detachment of fines by particle-wall collision. This mechanism also leads to attachment of fines to the wall.

3. Detachment of fines by fluid-flow.

4. Re-attachment of fines to a carrier particle due to air flow; fines which have been previously released in the air may re-attach to a carrier particle.

These four mechanisms are illustrated in Figure 5.

\section{Detachment of fines by particle-particle collisions}

The impact of a carrier particle with another particle or a wall will induce de-agglomeration or release of fines. ${ }^{22,43}$ Because four-way coupled CFD-DEM simulations have not been performed, this mechanism has previously not taken into account on the level of particle-particle collisions. As this work 
does take into account four-way coupling between the fluid and the particles, this mechanism will be taken into account. The collision of two carrier-particles which are coated with fines will cause release and exchange of some of these fines. The importance of this mechanism depends upon a number of factors, amongst which are the collision frequency of particles with other particles in the inhaler, and the normal impact velocity of the two particles undergoing the collision. The range of relative velocities of impact that are considered are between $0 \mathrm{~ms}^{-1}$ and $15 \mathrm{~ms}^{-1}$. This corresponds to the range of impact velocities in the simulation of the whole inhaler device, which is discussed later.

To simulate the process of fines release caused by the collisions of two carrier-particles a number of micro-scale simulations have been performed where an empty carrier particle (i.e. no fines attached) collides with a loaded carrier particle (i.e. completely filled with fines). In these simulations 2 parameters have been varied: the normal impact velocity, $U$, and the Hamaker constant, $H a$. In general, the larger the impact velocity and the lower the Hamaker constant, the more fines that are released during the collision. It was found that the rolling resistance model, as outlined previously, is an important mechanism that prevents the fines from rolling too easily over the carrier particle. Thus, including the resistance model makes it much more difficult for fine particles to detach. Figure 6 shows the results of the micro-scale simulation in terms of the percentage of fines released due to the collision as a function of normal impact velocity for various values of $H a$. 25 simulations for different velocities were performed for each Hamaker constant, thus totalling 100 simulations. However, each simulation is computationally inexpensive. A simple empirical correlation for the fraction of fines that is released is proposed as,

$$
\eta=A_{0} U^{A_{1}}
$$

where $A_{0}$ and $A_{1}$ are fit parameters that depend on the Hamaker constant. Optimal fit values are presented in Table 2. Figure 6 also presents the quality of the fit (dashed lines). It can be seen that there is some deviation between the computational results and the experimental fit for the curve with $H a=1.0 \times 10^{-20}$. The correlation is valid for relative normal impact velocities between $0 \mathrm{~ms}^{-1}$ and 
$15 \mathrm{~ms}^{-1}$.

\section{Detachment/re-attachment of fines by particle-wall collision}

Another mechanism by which the concentration of fines on a carrier particle can change, is due to a collision of the carrier particle with a solid wall. This mechanism has been previously studied. ${ }^{23,43}$ In these previous works ${ }^{23,43}$ the total dispersion of fine particles resulting from particle-wall impact was studied.

However, in the current work we consider three possible mechanisms for the fines to be released. Firstly, fine particles from the carrier particle can be released onto the wall. These fine particles then adhere to the wall by means of the van der Waals force. Secondly, fine particles from the carrier particle can release and disperse into the air. Thirdly, if fine particles are adhered to the wall from a previous encounter, these can be re-attached to the carrier particle. This will obviously depend on the concentration of fines which are adhered to the wall.

The release of fines from a carrier particle due to this mechanism was studied by performing a number of simulations where a coated carrier particle collides with a wall. For these simulations, the impact velocity, impact angle and the Hamaker constant have been varied. A total of 125 simulations were carried out. As for the carrier-carrier collisions, it was found that the rolling resistance model, as outlined previously, is an important mechanism that prevents the fines from rolling too easily over the carrier particle. Thus, including a rolling resistance model decreases the detachment rate of fine particles. It was also found that the release and re-attachment of fines depends solely on the normal impact velocity, and not on the tangential impact velocity. This was also foundby previous work. ${ }^{43}$

Figure 7 shows the relative number of fines released from the carrier particle after impact with a wall for various normal impact velocities, for a Hamaker constant of $1.0 \times 10^{-19} \mathrm{~J}$. There is a discontinuity in the number of released fines as a function of normal impact velocity with a wall. This 
discontinuity is caused by the rolling resistance: when fine particles can overcome the rolling resistance, meaning that their momentum exceeds the momentum required to overcome the local surface roughness, the detachment rate increases significantly. The normal impact velocity where the discontinuity occurs depends on the value of the Hamaker constant. At normal impact velocities below the discontinuity, the fine particles which are attached to the side of the carrier particle are affected by the impact, and can potentially detach. However, at normal impact velocities velocities which lie above the discontinuity, all fine particles are affected by the collision: even the fines on the top side of the carrier particle move and may be detached.

The fraction of particles that are detached from the carrier can be empirically expressed as

$$
\eta_{\text {detach }}= \begin{cases}B_{0} U_{n}^{B 1} & \text { if } U_{n}<B 2 \\ B_{3} U_{n}^{B 4} & \text { if } U_{n}>B 2\end{cases}
$$

and the corresponding empirical coefficients, for the various Hamaker constants are given in Table 3. This expression is valid for impact velocities between $0 \mathrm{~ms}^{-1}$ and $15 \mathrm{~ms}^{-1}$.

During the collision of the carrier particle with the wall, some of the fines are directly deposited onto the wall and stay attached on the wall, due to the adhesive nature of the fines. This will lead to fine particle retention in the inhaler device. Figure 8 shows the fraction of fines that is removed from the carrier particle which is directly deposited onto the wall, as a function of the normal impact velocity. Similar to the fraction of fines released from a carrier particle due to a wall collision, there is a discontinuity near a normal impact velocity of $U_{n} \approx 5 \mathrm{~ms}^{-1}$, because of the mechanism discussed earlier. The fraction of particles that are detached from the carrier can be empirically expressed as

$$
\eta_{\text {wall }}= \begin{cases}C_{0} U_{n}^{C 1} & \text { if } U_{n}<C 2 \\ C_{3} U_{n}^{C 4} & \text { if } U_{n}>C 2\end{cases}
$$


and the corresponding empirical coefficients, for the various Hamaker constants are given in Table 4 . When fine particles have been deposited on the wall from a previous collision event, these fines can be re-attached to a carrier particle during a subsequent collision event. To simulate the release of fines from a wall onto a colliding carrier particle, a number of simulations have carried out varying the concentration of fines attached to the wall and varying the concentration of fines on the carrier particle involved in the collision.

It was found, that the number of fines attached to the carrier particle involved in the wall collision does not play a role in this re-attachment process. Figure 9 shows the results of the actual number of fines that are removed from the wall as a function of the relative surface concentration of fines on the wall, for particles with $H a=1.0 \times 10^{-19} \mathrm{~J}$. The relative surface concentration is 1 if the wall is completely covered in the maximum, mono-disperse packing of particles. The fines that are removed from the carrier particle and deposited on the wall can be described satisfactorily with the empirical equation

$$
N_{\text {remove }}=D_{0} c_{\text {wall }}^{\text {1 }}
$$

where $c_{w a l l}$ is the concentration of fine particles at the wall, in number of particles per $\mu m^{2}$, and $N_{\text {remove }}$ is the number of fine particles released from the wall and re-attached to the colliding carrier particle The empirical values for $D_{0}$ and $D_{1}$ for various Hamaker values are given in Table 5 .

\section{Detachment of fines by fluid-flow}

An additional mechanism by which fines are removed from the carrier particle, is by the interaction of the carrier particle and the fluid flow. This mechanism has been reported in previous work. ${ }^{21,22}$ This micro-model mechanism is the only mechanism considered in Cui $e t a l^{21}$ and has been also extensively studied by Calvert et $a l .{ }^{44}$

If the DEM model is considered excluding the rolling resistance, our micro-model findings are very similar to previous work. ${ }^{21}$ In total, 10 simulations for each value of the Hamaker constant have been 
carried out, thus totalling 40 simulations. The mechanism of the fines detaching from the carrier is predominantly caused by the fines rolling over the carrier particle prior to detaching. This can also be seen from the figures presented by Cui et al. ${ }^{21}$ The fines are relatively easily removed from the carrier particle and the fraction of fines that is removed per unit time can be described by the empirical equation

$$
\eta=E_{0}\left(U_{f}-U_{0}\right)^{E 1}
$$

when the relative fluid velocity exceeds that of a certain value, $U_{0}$. The values for $E_{0}$ and $E_{1}$, as well as the relative fluid velocity which the equation becomes applicable, are given Table 6 .

However, in a real situation, the fines will experience a significant rolling resistance due to the surface roughness and their non-spherical nature. Our findings show that if rolling resistance in enabled, as described previously, the detachment of fines by the fluid flow is negligible in the regime studied (i.e. relative fluid velocities of up to $20 \mathrm{~ms}^{-1}$ ).

\section{Re-attachment of fines by particle moving through the fluid}

As carrier particles travel through the inhaler, they may encounter fines which have been previously detached. The number of fines re-attached to the carrier will strongly depend upon the local volume fraction of fines, as well as the difference in velocity between the fines and the carrier particle. A number of simulations with un-coated carrier particles have been performed in environments with varying volume fractions of fines.

The results of 20 simulations carried out show that the number of re-attached fines is close to what would be obtained using a simple model which assumes that the carrier particle travels through a cylinder with the cross-sectional area given by the cross sectional area of the carrier particle, and the height given by $V \cdot \Delta t$, where $V$ represents the relative velocity between the carrier particle and the 
fines. This gives, for the number of fines reattaching per unit time

$$
N_{f}=\frac{6 d^{2} V \Delta t \alpha_{\gamma}}{d_{f}^{3}}
$$

where $\alpha_{\gamma}$ is the local volume fraction of fines. The results are almost independent of Hamaker constant.

\section{Simulation set-up and domain}

The fitted micro-models, as detailed in the previous section, are applied to a realistic model geometry of a prototype inhaler. Discrete element model (DEM) particles represent the individual carrier particles, which are modelled as point particles coupled to the fluid momentum equations. ${ }^{45}$

In addition to the equations for the fluid and particle phases presented previously, two additional passive scalars are introduced to account for the fines. The passive scalar defined for each particle represents the number of fines relative to the initial number of fines. The passive scalar that is tracked with the fluid velocity represents the volumetric concentration of fines. The passive scalar equation which is solved for in the fluid reads

$$
\frac{\partial \alpha \rho \gamma}{\partial t}+\frac{\partial}{\partial x_{j}}\left(\alpha \rho u_{f, j} \gamma\right)=\frac{\partial}{\partial x_{j}} \alpha \Gamma \frac{\partial \gamma}{\partial x_{j}}+S_{\gamma}
$$

where $\gamma$ represents the concentration of fines, $\alpha$ the local fluid volume fraction, and $\Gamma$ the diffusivity of the scalar representing the concentration of fines. The value for the local diffusivity is taken from the LES closure model. Finally, $S_{\gamma}$ represents the local sinc or source terms arising from the exchange of fines, as prescribed by the micro models outlined in the previous sections. The contributions to this source term can be written out as

$$
S_{\gamma}=S_{\gamma}^{P P}+S_{\gamma}^{P W}+S_{\gamma}^{\text {Flow }}+S_{\gamma}^{\text {Recapture }}
$$


where the right hand side contributions are the source terms from the particle-particle collisions, the particle-wall collisions, detachment due to the air flow, and recapture of fines, respectively. These source terms are given in terms of concentration per unit time, i.e. the number of released or recaptured fines per unit volume per unit time. The source terms are determined from the micro-models as they occur in a particle time-step, for the collisional contributions, or in a fluid time-step for the flow interaction phenomena.

\section{Geometry and Mesh}

The complete framework is tested on a prototype inhaler device geometry, the screen-haler, which is basically an L-shaped cylindrical channel. Such simple geometries have been used in order to facilitate elucidation of the role of the formulation. ${ }^{46,47}$ A sketch of the geometry is shown in Figure 10. The length of the inhaler, $L$, is $45 \mathrm{~mm}$, the diameter of the inlet and outlet, $D$, is $7.0 \mathrm{~mm}$, and the height $H$ is $16 \mathrm{~mm}$.

Two computational meshes are used to carry out the single-phase simulations in order to show that the solution is grid independent. The coarse geometry is then used to carry out the gas-particle simulations. The coarse mesh contains a total of 870,000 computational cells and the finer mesh contains a total of 1,299,000 computational cells. The refinement is achieved by refining the nodal spacing equally in all directions. Both meshes resolve the wall boundary layer, and contain 5 and 12 mesh points within the $y^{+}=10$ layer, respectively. The finest mesh cell has the width of approximately a single carrier particle.

\section{Initial Conditions}

Initially, the fluid in the inhaler is at rest. The bottom center of a dense packed pile of particles is placed at a distance $l=39 \mathrm{~mm}$ upstream of the outlet, see figure 10. The dense pack of particles is formed by a separate DEM simulation of 13,000 particles falling into the downward facing point of a 
square pyramid, and forming a packing with a particle volume fraction of 0.69 in that geometry. This separate DEM simulation was run until all the particles had a negligible velocity, which corresponded to 0.2 seconds of physical time.

The scalar representing the concentration of fines, $\gamma$, is initially set to zero everywhere throughout the domain.

\section{Boundary Conditions}

The flow-rate through the inhaler varies as a function of time, and is taken from a typical patient curve $^{48,49}$ The curve used in this study is shown in Figure 12, which shows the flow rate as a function of time. Figure 12 also shown that the peak flow rate is reached after approximately $0.3 s$. This curve is used as the boundary condition at the inlet of the inhaler. The boundary condition is specified as a flat velocity profile. The mass flow is thus equally spread over the inhaler inlet. At the outlet, as pressure boundary is specified, setting the fixed value for the atmospheric pressure. A no-slip wall condition for the fluid velocities is enforced on all walls. This is suitable, as the boundary layer along the wall is resolved using DNS.

\section{Simulation Framework}

The discretisation of the Navier-Stokes equations is done using a finite volume approach, combined with a second order accurate three point backward Euler time discretisation for the temporal terms and a second order accurate central differencing scheme for the advection term. The pressure velocity coupling is done in a fully coupled framework, using one outer iteration per time-step. ${ }^{50,51}$

\section{Results and Discussion}

The simulations were performed on the HPC facility of Imperial College London, using 16 cores per simulation. The simulations took approximately 6 hours of computational time on 16 cores for 0.2 seconds of real time simulation. This corresponds to the time that is required for all of the carrier 
particles to leave the prototype inhaler device. There are two integration time-steps required for the simulation, the time-step associated with the fluid equations and the time-step associated with the particle motion. Both time-steps are dynamically varied, based upon the CFL number and the velocity difference between the particles respectively. The time-steps were on the order of $1.0 \cdot 10^{-5} \mathrm{~s}$ for the fluid, and $1.0 \cdot 10^{-8} s$ for the particle phase. The particle-time step is also governed by the particle properties; how larger the Young's modulus of the material, how smaller the time-step required to resolve the collision. ${ }^{52}$

The emptying time of an inhaler will strongly depend on the type of the inhaler, the formulation used and the patient inhalation curve. For instance, longer emptying times are expected when capsule devices are used ${ }^{53}$ than in the current case. The results show that it takes approximately $0.12 s$ of physical time for all the carrier particles to leave the domain which is considered in this study. This is defined as the emptying time.

Figure 13 shows four snapshots of the air velocity and the location of the carrier particles at different times. The flow enters homogeneously from the top of the domain, and is initially laminar. It can be seen that the flow velocity increases as a function of time, becomes increasingly turbulent, and that the carrier particles start to move with the flow after about $0.02 \mathrm{~s}$. Although the majority of the carrier particles directly move towards the exit of the inhaler, there is a significant part of the carrier particles which stays behind in the bend of the inhaler and take a significantly longer time to leave. These particles stay behind because they are caught in a recirculating region that is established in the bend. This is because the detachment of the boundary layer on the inside of the bend creates a recirculation eddy on the other side, in the outside of the bend. This effect is common for high Reynolds number flows through a bend. ${ }^{54}$

It should be noted that all the particles have left the domain after just $0.12 \mathrm{~s}$. This is before the flow velocity has reached half of its maximum, as can be seen from Figure 12. 
Figure 14 shows the number of carrier particles (red line) and the number of fines that have detached from the carrier particle (blue line) that have left the inhaler as a function of time, for the fines with $H a=1.0 \cdot 10^{-20} \mathrm{~J}$. It can be clearly seen that the detached fines start leaving the inhaler around $0.03 s$, which is significantly earlier than the carrier particles start leaving the domain, around 0.055s. This is because the detached fines have a much lower inertia than the carrier particles. The fines detached in the early stage of the inhalation process therefore reach the exit before any of the carrier particles. All the detached fines have left the inhaler at $0.10 \mathrm{~s}$ and all the carrier particles have left at $0.12 s$.

Figure 15 shows the number of fines exiting the inhaler that are detached from the carrier particle as a function of time for various values of Hamaker constant of the fines. The value of the Hamaker does not have an effect on the start and end times of the fines exiting the inhaler, only on the total number of detached fines vs attached fines leaving the inhaler. The results show an approximate relative decrease of $25 \%$ in the detachment of fines if the Hamaker constant is 5 times as large.

Figure 16 shows the number of fines exiting the inhaler that are still attached to the carrier particle as a function of time, for the various values of Hamaker constant. Since the fines are attached to the carrier particles, the start and end times of the attached fines exiting the inhaler are governed by the dynamics of the carrier particles and do not depend on the value of the Hamaker constant. The results show a gradual increase in the number of fines attached as the Hamaker constant is increased.

The importance of the micro-model mechanism which describes the detachment of fine particles from the carrier particle due to the direct interaction with air, as is described previously, has been investigated by comparing a simulation including the mechanism with one that excludes it. Figure 17 shows the fines exiting the inhaler as a function of time, either detached or attached to a carrier particle, comparing these two simulations, with $H a=5.0 \cdot 10^{-19} \mathrm{~J}$. For this value of Hamaker, it can be seen 
the results are nearly the same, and that the blow-off mechanism is not important for this value of Hamaker constant. This is in contrast of what has been suggested previously in the literature. ${ }^{21,44}$ However, it should be noted that for the current type of inhaler, the local fluid velocity does not exceed around $15 \mathrm{~m} / \mathrm{s}$ within the first $0.12 \mathrm{~s}$. Therefore, it may be that this mechanism is more important for other types of inhalers, i.e. inhalers where the local fluid velocity becomes very large.

When carrier particles collide with the wall, some of the detached fines may directly adhere to the wall. This mechanism was described in a previous section. The fines that are adhered to the wall may, due to a subsequent collision with a carrier particle and the wall, be detached from the wall and re-dispersed into the air flow or picked up by the carrier particle. Figure 18 shows the relative concentration of fine particles adhered to the wall at the end of the simulation. It can be seen that most of the fines adhere to the wall in two locations: near the initial position of the carrier particles and in a position slightly downstream from this initial position. Near the initial position of the carrier particles, collisions with the wall are very frequent - although impact velocities are generally small. After the onset of movement of the carrier particles, many of them will impact the wall due to gravity slightly downstream of the initial position. The collisions are less frequent, but will typically have significantly higher impact velocity. More quantitative results are presented in Table 7 . This table shows the percentage from the total initial number of fines which are adhered to the wall and the percentage which are detached from the carrier particle and leave the domain individually. A very strong effect of the Hamaker constant is observed.

It should be noted that the so-called retainment of fines will strongly depend on the geometry of the inhaler, the fluid flow development in the inhaler, the initial position of the carrier particles, and the direction of gravity with respect to the inhaler. Since the retainment is due to particle-wall collisions and depends on the particle-wall interaction, this prediction will obviously also depend on the properties of the wall. Nevertheless, from a device development perspective the prediction is of interest since a spatially resolved measure of the amount of fines on the wall can be obtained experimentally (using e.g. 
spectroscopic techniques). This prediction could thus be used to at least qualitatively verify the model predictions and to also suggest changes to the device geometry that would decrease the retainment. Obviously, the underlying micro-model could also suggest changes to the formulation that would decrease the retainment.

Figure 19 shows the time-averaged contribution of particle-wall collisions on the release of fines into the air flow. The mechanism of particle-wall collisions leading to the detachment of fines has three regions. The first region is around the initial position of the carrier particles, where the particle-wall collisions are frequent but have a low impact velocity. The second region is slightly further downstream than the initial position, where carrier particles collide with the wall after the onset of initial movement. Although the kinetic energy of these impacts is typically larger than in the previous region, it is still relatively small. These two regions are also present in the mechanism for fine retainment, as described previously. The third region concerns isolated small areas around the inhaler. These small areas show where a single or a limited number of carrier particles have collided with a large impact velocity. Because of the limited number of carrier particles that collides, this does not seem to be important for fines retainment. However, because of the high impact velocity, it does mean that all of the attached fines are detached during the violent collision process.

Figure 20 shows the time-averaged relative contribution of particle-particle collisions to the release of fines to the air. The top image of Figure 20 shows a cross section. It can be clearly seen that most particle-particle collisions occur close to the bottom wall, predominantly fairly near the initial location of the particles. Once the particles have traveled further downstream, they become more dispersed the probability of particle-particle collisions is reduced. This mechanism is proportional to the local volume fraction of the carrier particles and the velocity difference between the carrier particles (their so-called granular temperature). The local volume fraction is particularly large when the carrier particles are near their initial position. The granular temperature becomes significant further downstream. These 
two effects can be clearly identified in the figure.

Figure 21 shows the time-averaged combined effect of all mechanisms to the contribution of detachment of fine particles from carrier particles. Most of the detachment occurs early on in the inhalation process and near the bottom wall of the inhaler. The particle-particle interactions are the biggest contribution to the release of fines (around 75\%), although initially the particle-wall collisions are also significant.

\section{Conclusions}

In this paper a comprehensive model is constructed to predict the behaviour of a dry powder inhaler, including the carrier particles which are coated with small fine drug particles and their interaction with turbulent air flow. The developed model comprises a multi-scale approach, where the phenomena in a dry powder inhaler are studied at the micro, meso and macro scales. The model thus provides a link between the properties of particles and the performance of the inhalation device.

At the micro-scale, the interactions of the small active drug particles, fines with a diameter of $2 \mu m$, and the carrier particle, with a diameter of $70 \mu m$ are studied to capture the behaviour of four different mechanisms leading to the release or re-attachment of the fines: carrier-carrier particle collisions, carrier particle-wall collisions, detachment of fines by carrier particle-air interactions, and re-attachment of fines resulting from pick-up onto the carrier particle. The micro-scale study has lead to coarse-grained models describing the interaction of the small active drug particles. These coarse-grained models can be used to determine the behaviour of fines at larger scales.

At the meso-scale the larger carrier particles (diameter $\approx 70 \mu m$ ), and all of their interactions, are 
modelled individually using discrete element model (DEM). Large eddy simulations (LES) are used to model the turbulent nature of the air flow in the inhaler. Finally, at the macro-scale, simulations of the complete inhaler are carried out. The inhalation process of one dosage from a prototype inhaler using a patient relevant air flow profile are simulated, and the subsequent detachment of fines is studied.

The results at the macro-scale show that the fines start leaving the inhaler before the carrier particles. The fines leave the inhaler before the carrier particles because they accelerate faster due to their smaller inertia. The results on the macro-scale also show that the total release efficiency of the fine particles is between $10 \%$ and $30 \%$, depending on the Hamaker constant of the particles, using typical settings for the properties of both particle types. These values for release efficiency are in good agreement what is typically found experimentally. ${ }^{47}$ It is also shown that carrier particle-particle interactions at the beginning of the inhalation process are the biggest contributor to the detachment of fines, followed by the carrier-particle wall interactions. The factors behind this are the local volume fraction of the carrier particles and the granular temperature of the carrier particles, as these two determine the frequency and effect of collisions. The detachment of fines in the prototype inhaler directly by air flow is shown to be negligible. Finally, the location and relative concentration of retained fines on the wall segments of the inhaler is shown.

The developed framework can be thus be used to predict powder emptying and dispersion as well as retention in a dry powder inhaler. In the present work the models were used to make predictions for a prototype inhaler, but the same type of modeling can clearly be used to make predictions for any other device or for powders with different properties. The model is thus a very valuable tool for device development to make sure that the device is suitable for the particular formulation. Since the model can incorporate any inhalation profile, it can also be used to analyse whether a device is suitable for a particular patient group.

In addition to providing a link between the properties of particles and the performance of the inhalation 
device, the models at the different levels are useful in their own right. For example,the micro models can be used to study how the release of fines depends on the properties of the particles, such as their stiffness, adhesive and cohesive interactions. In addition, more elaborate models can be developed in a similar way, to address the issue of size and shape so that effects of shape can be studied.

Although it was beyond the scope of the present work, this type of modeling can also be extended to study formulation processing to understand how the method of depositing the particles on the carriers affects the distribution of fines on the carrier particles. The framework presented in this paper obviously requires experimental validation. As already noted, we have taken steps in this direction. To this end is should be noted that direct validation of particle motion in a device is very challenging as it requires experimental techniques with a very high spatial and temporal resolution. In addition, the detailed particle trajectories also depend on the initial configuration which is not always easy to control. In any case, we hope that the present study will serve to motivate future work in this area.

\section{References}

${ }^{1}$ Derjaguin B, Muller V, Toporov Y. Effect of contact deformations on the adhesion of particles. Journal of Colloid and Interface Science. 1975;53(2):314-326.

${ }^{2}$ Islam N, Gladki E. Dry powder inhalers (DPIs)-A review of device reliability and innovation. International Journal of Pharmaceutics. 2008;360(1-2):1-11.

${ }^{3}$ de Boer AH, Chan HK, Price R. A critical view on lactose-based drug formulation and device studies for dry powder inhalation: Which are relevant and what interactions to expect? Advanced drug delivery reviews. 2012;64(3):257-74.

${ }^{4}$ Grasmeijer F, Grasmeijer N, Hagedoorn P, Frijlink HW, de Boer A. Recent Advances in the Fundamental Understanding of Adhesive Mixtures for Inhalation. Current Pharmaceutical Design. 2015; $21: 5900-5914$. 
${ }^{5}$ Hassoun M, Ho S, Muddle J, Buttini F, Parry M, Hammond M, Forbes B. Formulating powder-device combinations for salmeterol xinafoate dry powder inhalers. International Journal of Pharmaceutics. $2015 ; 490(1-2): 360-7$.

${ }^{6}$ Steckel H, Mueller B. In vitro evaluation of dry powder inhalers I: Drug deposition of commonly used devices. International Journal of Pharmaceutics. 1997;154:19-29.

${ }^{7}$ Steckel H, Markefka P, TeWierik H, Kammelar R. Effect of milling and sieving on functionality of dry powder inhalation products. International Journal of Pharmaceutics. 2006;309(1-2):51-59.

${ }^{8}$ Shur J, Saluja B, Lee S, Tibbatts J, Price R. Effect of Device Design and Formulation on the In Vitro Comparability for Multi-Unit Dose Dry Powder Inhalers. The AAPS Journal. 2015;17(5).

${ }^{9}$ Ooi J, Traini D, Hoe S, Wong W, Young PM. Does carrier size matter? A fundamental study of drug aerosolisation from carrier based dry powder inhalation systems. International journal of pharmaceutics. 2011;413(1-2):1-9.

10 Tuli R, George G, Dargaville T, Islam N. Studies on the Effect of the Size of Polycaprolactone Microspheres for the Dispersion of Salbutamol Sulfate from Dry Powder Inhaler Formulations. Pharmaceutical Research. 2012;29(9):2445-2455.

${ }^{11}$ Young PM, Wood O, Ooi J, Traini D. The influence of drug loading on formulation structure and aerosol performance in carrier based dry powder inhalers. International Journal of Pharmaceutics. 2011;416(1):129-135.

${ }^{12}$ Weiss C, McLoughlin P, Cathcart H. Characterisation of dry powder inhaler formulations using atomic force microscopy. International Journal of Pharmaceutics. 2015;494(1):393-407.

${ }^{13}$ Kubavat Ha, Shur J, Ruecroft G, Hipkiss D, Price R. Influence of primary crystallisation conditions on the mechanical and interfacial properties of micronised budesonide for dry powder inhalation. International Journal of Pharmaceutics. 2012;430(1-2):26-33. 
${ }^{14}$ Grasmeijer F, Hagedoorn P, Frijlink HW, de Boer AH. Drug content effects on the dispersion performance of adhesive mixtures for inhalation. PloS one. 2013;8(8):e71339.

${ }^{15}$ Grasmeijer F, Lexmond AJ, van den Noort M, Hagedoorn P, Hickey AJ, Frijlink HW, de Boer AH. New mechanisms to explain the effects of added lactose fines on the dispersion performance of adhesive mixtures for inhalation. PloS one. 2014;9(1):e87825.

${ }^{16}$ Islam N, Stewart P, Larson I, Hartley P. Lactose surface modification by decantation: Are drug-fine lactose ratios the key to better dispersion of salmeterol xinafoate from lactose-interactive mixtures? Pharmaceutical Research. 2004;21(3):492-499.

17 Jones MD, Santo JGF, Yakub B, Dennison M, Master H, Buckton G. The relationship between drug concentration, mixing time, blending order and ternary dry powder inhalation performance. International Journal of Pharmaceutics. 2010;391(1-2):137-147.

18 Jaffari S, Forbes B, Collins E, Barlow DJ, Martin GP, Murnane D. Rapid characterisation of the inherent dispersibility of respirable powders using dry dispersion laser diffraction. International Journal of Pharmaceutics. 2013;447(1-2):124-131.

${ }^{19}$ Edwards D. Delivery of biological agents by aerosols. AIChE Journal. 2002;48(1):2-6.

${ }^{20}$ Weers JG, Miller DP. Formulation Design of Dry Powders for Inhalation. Journal of Pharmaceutical Sciences. 2015;104(10):3259-3288.

${ }^{21}$ Cui Y, Schmalfuss S, Zellnitz S, Sommerfeld M, Urbanetz N. Towards the optimisation and adaptation of dry powder inhalers. International Journal of Pharmaceutics. 2014;470(1-2):120-132.

${ }^{22}$ Yang J, Wu CY, Adams M. Numerical Modelling of Agglomeration and Deagglomeration in Dry Powder Inhalers : A Review. Current Pharmaceutical Design. 2015;21:1-8.

23 Tong Z, Kamiya H, Yu A, Chan HK, Yang R. Multi-Scale Modelling of Powder Dispersion in a Carrier-Based Inhalation System. Pharmaceutical research. 2014;32(10):13-15. 
${ }^{24}$ Germano M, Piomelli U, Moin P, Cabot WH. A dynamic subgrid-scale eddy viscosity model. Physics of Fluids A. 1991;3(7):1760-1765.

${ }^{25}$ Mallouppas G, van Wachem B. Large Eddy Simulations of Turbulent Particle-Laden Channel Flow. International Journal of Multiphase Flow. 2013;54:65-75.

${ }^{26}$ Maxey MR, Riley JRJ. Equation of motion for a small rigid sphere in a nonuniform flow. Physics of Fluids. 1983;26(4):883.

${ }^{27}$ Wen CY, Yu YH. A generalized method for predicting the minimum fluidization velocity. AIChE Journal. 1966;12(3):610-612.

${ }^{28}$ Rowe PN. Drag forces in a hydraulic model of a fluidized bed, part II. Trans Inst Chem Engs. 1961; $39: 175-180$

${ }^{29}$ Allen MP, Tildesley DJ. The Computer Simulation of Liquids, Vol 42. Oxford University Press. 1989.

${ }^{30}$ Cundall P, Strack ODL. A discrete numerical model for granular assemblies. Géotechnique. 1979; $29(1): 47-65$.

31 Johnson PC, Nott P, Jackson R. Frictional-collisional equations of motion for particulate flows and their application to chutes. Journal of Fluid Mechanics. 1990;210:501-535.

${ }^{32}$ Tsuji Y, Tanaka T, Ishida T. Lagrangian numerical simulation of plug flow of cohesionless particles in a horizontal pipe. Powder Technology. 1992;71(3):239-250.

${ }^{33}$ Anand A, Curtis JS, Wassgren CR, Hancock BC, Ketterhagen WR. Predicting discharge dynamics of wet cohesive particles from a rectangular hopper using the discrete element method (DEM). Chemical Engineering Science. 2009;64(24):5268-5275.

34 Tomas J. Adhesion of ultrafine particles-Energy absorption at contact. Chemical Engineering Science. $2007 ; 62(7): 5925-5939$. 
${ }^{35} \mathrm{Ai}$ J, Chen JF, Rotter JM, Ooi JY. Assessment of rolling resistance models in discrete element simulations. Powder Technology. 2011;206(3):269-282.

${ }^{36}$ Mindlin RD, Deresiewicz H. Elastic spheres in contact under varying oblique forces. J of Appl Mech. $1953 ; 20: 327-344$.

37 Johnson KL. Contact Mechanics. Cambridge, UK: Cambridge University Press. 2003.

${ }^{38}$ Castellanos A. The relationship between attractive interparticle forces and bulk behaviour in dry and uncharged fine powders. Advances in Physics. 2005;54(4):263-376.

${ }^{39}$ Sagaut P. Large Eddy Simulation for Incompressible Flows. Springer, 3rd ed. 2005.

${ }^{40}$ Pope SB. Turbulent Flows. Cambridge: Cambridge Univ Press, 6th ed. 2000.

${ }^{41}$ Lilly DK. A proposed modification of the Germano subgrid scale closure method. Physics of Fluids A. 1992;4(3):633-635.

42 Tsukada M, Irie R, Yonemochi Y, Noda R, Kamiya H, Watanabe W, Kauppinen EI. Adhesion force measurement of a DPI size pharmaceutical particle by colloid probe atomic force microscopy. Powder Technology. 2004;141(3):262-269.

${ }^{43}$ Yang J, Wu CY, Adams M. DEM analysis of the effect of particle-wall impact on the dispersion performance in carrier-based dry powder inhalers. International journal of pharmaceutics. 2015; $487(1-2): 32-8$.

${ }^{44}$ Calvert G, Hassanpour A, Ghadiri M. Mechanistic analysis and computer simulation of the aerodynamic dispersion of loose aggregates. Chem Eng Res Des. 2011;89(5A):519-525.

${ }^{45}$ Crowe CT, Sharma MP, Stock DE. The Particle-Source-In Cell (PSI-CELL) Model for Gas-Droplet Flows. Journal of Fluids Engineering. 1977;99(2):325-333.

${ }^{46}$ Steckel H, Bolzen N. Alternative sugars as potential carriers for dry powder inhalations. International Journal of Pharmaceutics. 2004;270(October 2003):297-306. 
${ }^{47}$ Thalberg K, Berg E, Fransson M. Modeling dispersion of dry powders for inhalation. The concepts of total fines, cohesive energy and interaction parameters. International Journal of Pharmaceutics. 2012;427(2):224-33.

${ }^{48}$ Olsson B, Asking L. Critical aspects of the function of inspiratory flow driven inhalers. Journal of Aerosol Medicine. 1994;7(Suppl 1):S43-7.

${ }^{49}$ Delvadia RR, Wei X, Longest PW, Venitz J, Byron PR. In vitro tests for Aerosol Deposition. IV: Simulating Variations in Human Breath Profiles for Realistic DPI Testing. Journal of Aerosol Medicine and Pulmonary Drug Delivery. 2015;28(0):jamp.2015.1215.

${ }^{50}$ Denner F, van Wachem B. Fully-coupled balanced-force VOF framework for arbitrary meshes with least-squares curvature evaluation from volume fractions. Numerical Heat Transfer Part B: Fundamentals. 2014;65(3):218-255.

${ }^{51}$ van Wachem B, Mallouppas G, Denner F, Evrard F, Bartholomew P. http://www.multiflow.org/. 2016.

${ }^{52}$ Wilson R, Dini D, van Wachem B. A Numerical Study Exploring the Effect of Particle Properties on the Fluidization of Adhesive Particles. AIChE Journal. 2016;62(5):1467-1477.

${ }^{53}$ Behara SRB, Kippax P, Larson I, Morton DaV, Stewart P. Kinetics of emitted mass-A study with three dry powder inhaler devices. Chemical Engineering Science. 2011;66(21):5284-5292.

${ }^{54}$ Panton R. Incompressible Flow. Wiley, 4th ed. 2013.

${ }^{55}$ Perkins M, Ebbens SJ, Hayes S, Roberts CJ, Madden CE, Luk SY, Patel N. Elastic modulus measurements from individual lactose particles using atomic force microscopy. International Journal of Pharmaceutics. 2007;332(1-2):168-175.

${ }^{56}$ Sandell E. Industrial Aspects of Pharmecuticals. Swedish Pharmaceutical Press, CRC Press. 1992. 


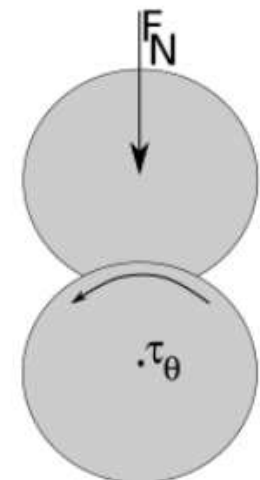

(a)

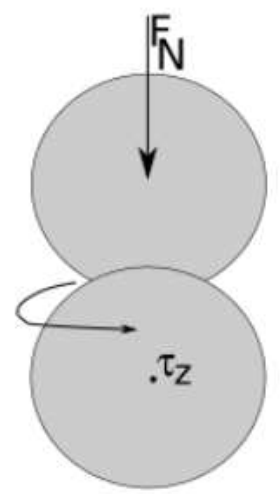

(b)

Figure 1: A sketch of the torque arising from: (a) rolling and (b) torsion. 


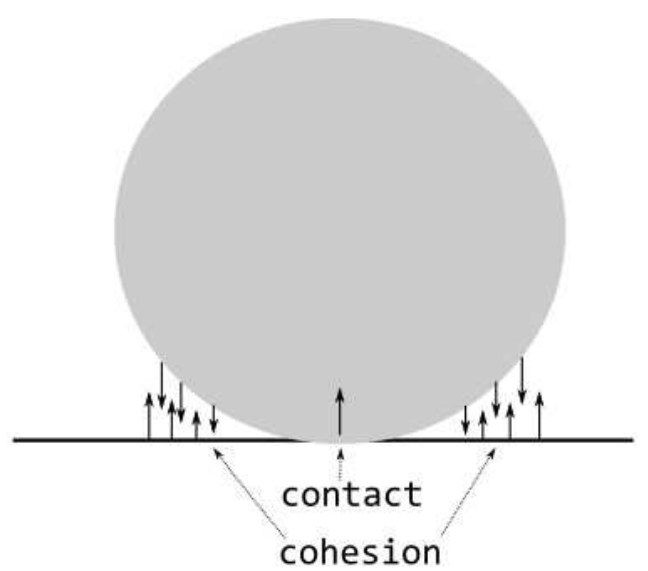

Figure 2: A sketch of the $\mathrm{DMT}^{1}$ adhesion model: a contact point which results in an elastic contact force, and the adhesion force outside of the direct contact point. 


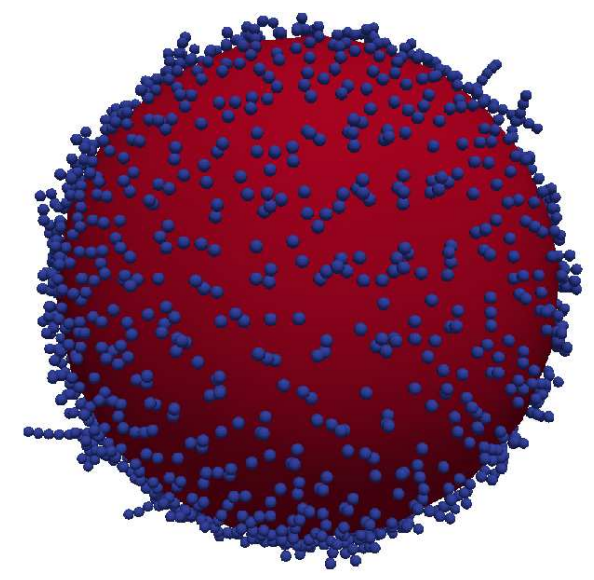

Figure 3: The result of a carrier particle coated with the 2,000 fine particles. 


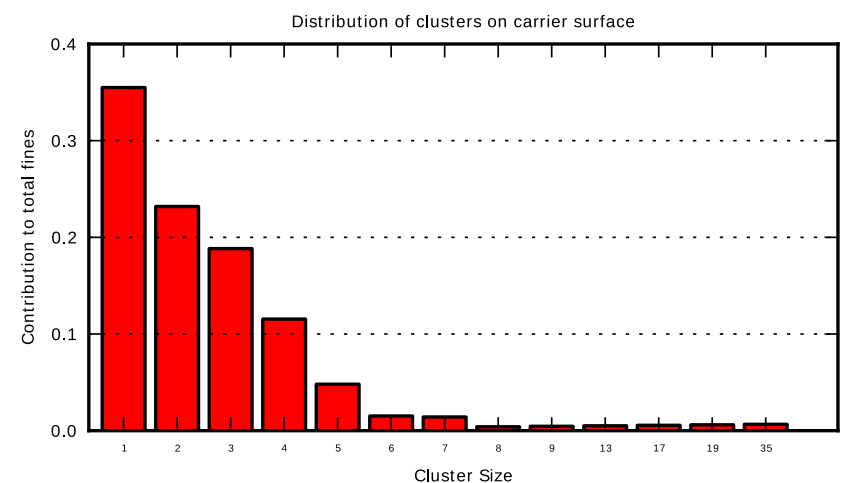

Figure 4: The distribution of clusters of fines on the carrier particle surface. 


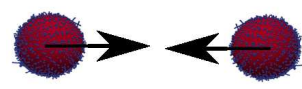

1

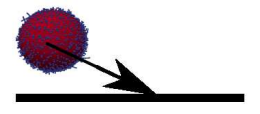

2

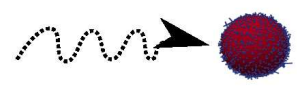

3

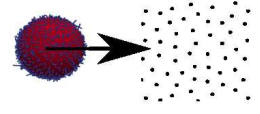

4

Figure 5: The four "micro-model" mechanisms studied in the present work: 1. Particle-particle collision, 2. Particle-wall collision, 3. Detachment of particles by fluid flow, and 4. re-attachment of fines. 


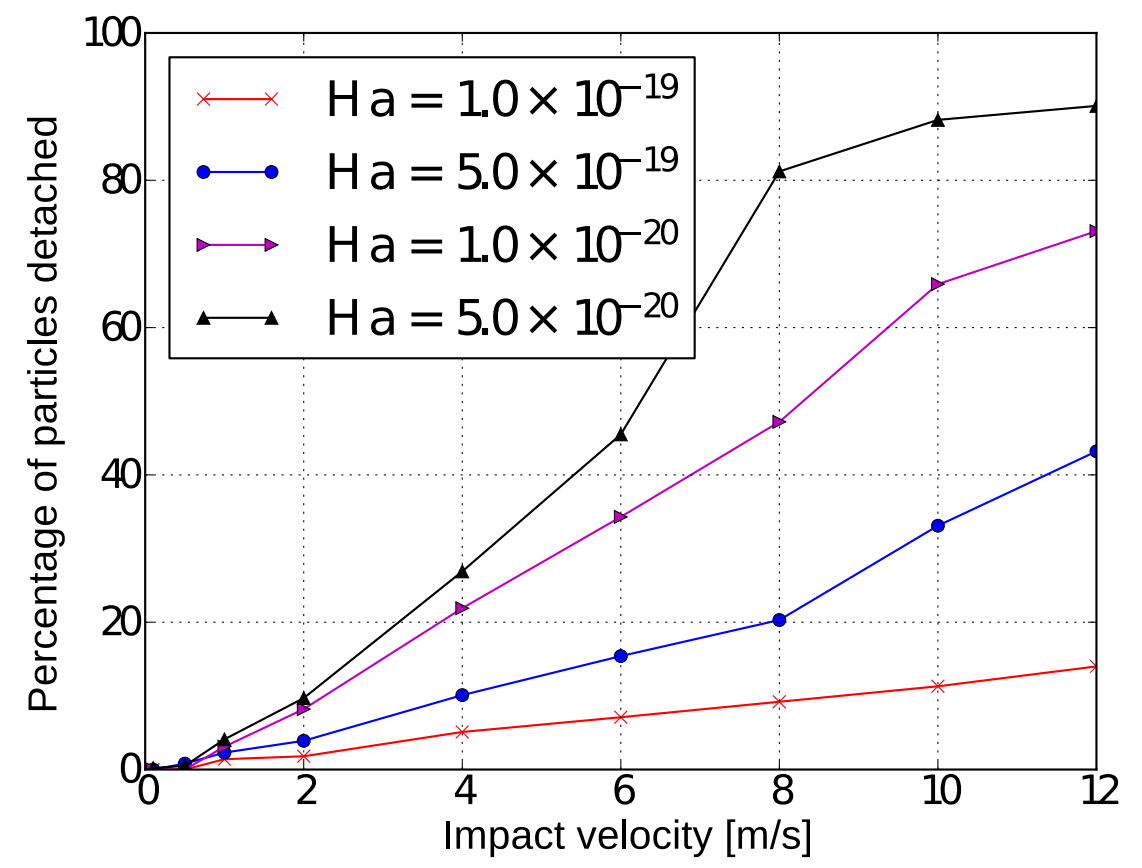

Figure 6: The percentage of small particles removed as a function for normal impact velocity for various $\mathrm{Ha}$ constants. The dashed lines represent the results predicted by Equation 40 . 


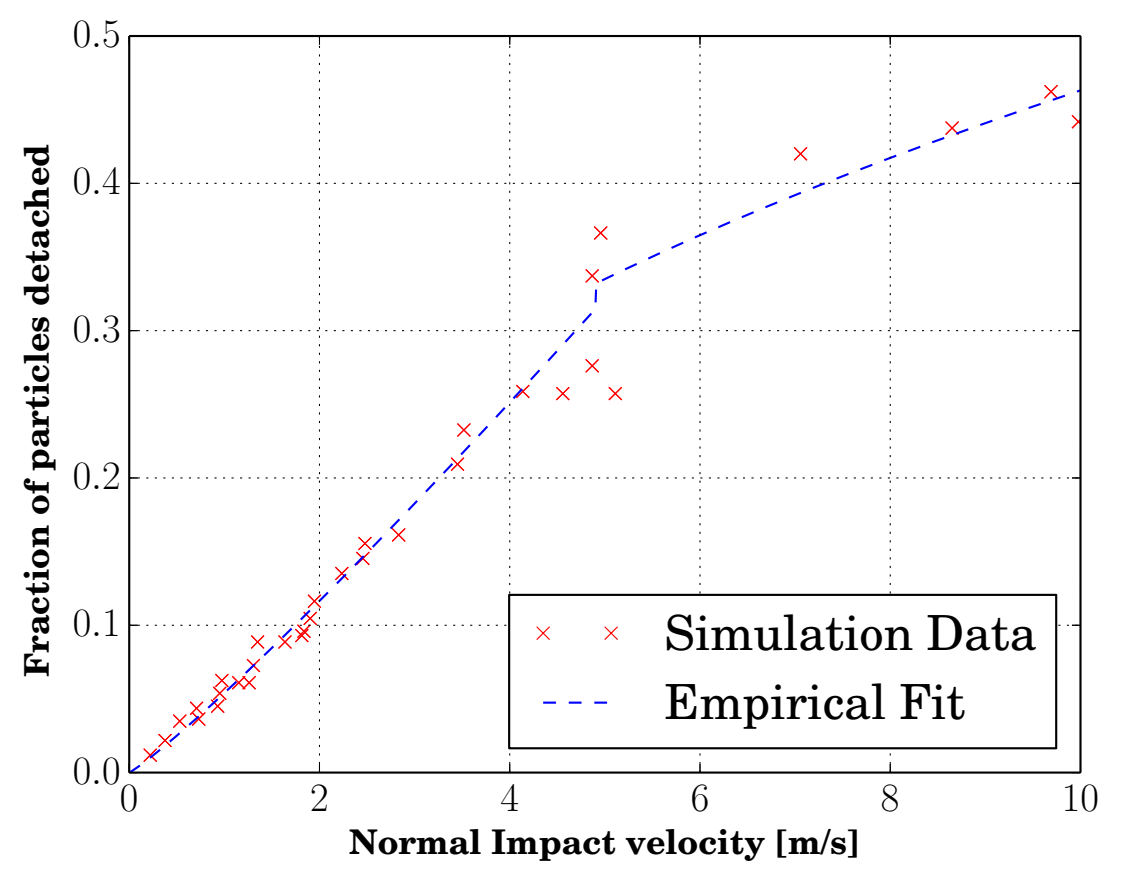

Figure 7: The simulation results (symbols) and the empirical fit for the fraction of fine particles that are removed from the carrier particle for $H a=1.0 \times 10^{-19} \mathrm{~J}$ as a function of normal impact velocity with a wall. 


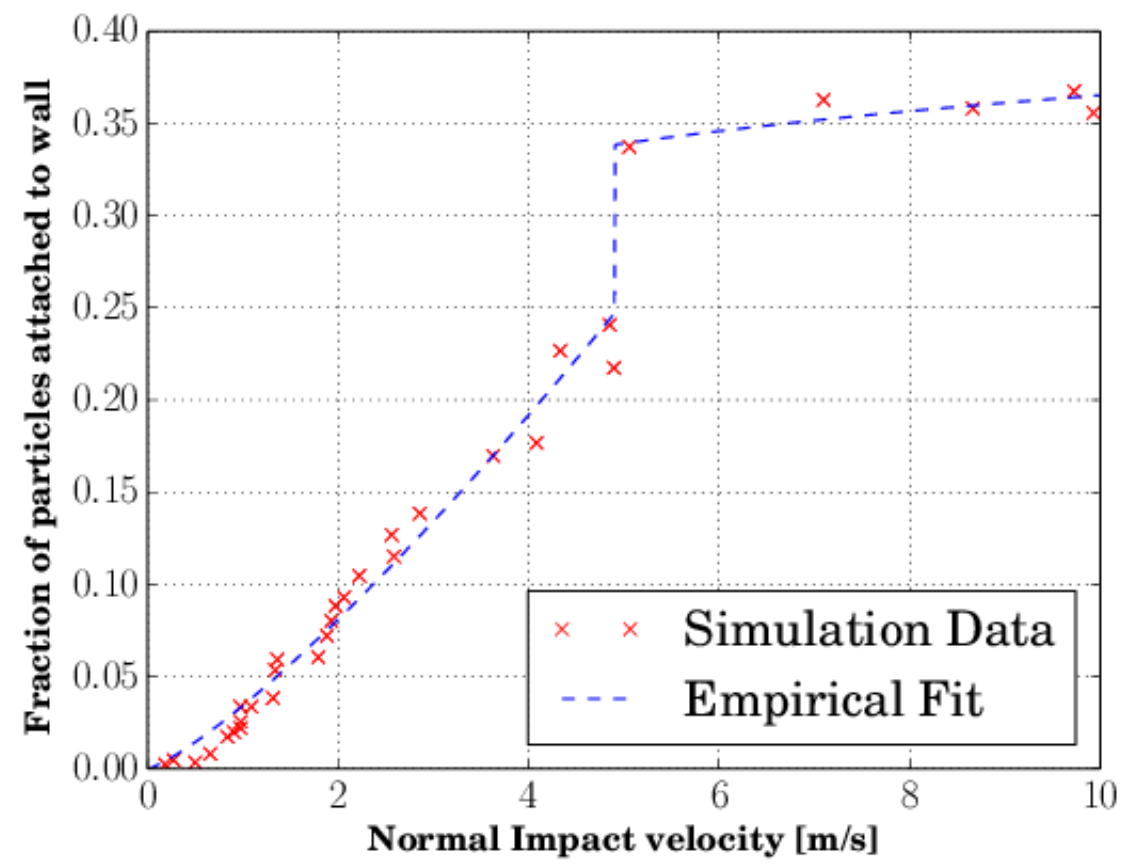

Figure 8: The simulation results (symbols) and the fit for the fraction of fine particles that are removed from the carrier particle and deposited onto the wall for $H a=1.0 \times 10^{-19} \mathrm{~J}$ as a function of normal impact velocity with a wall. 


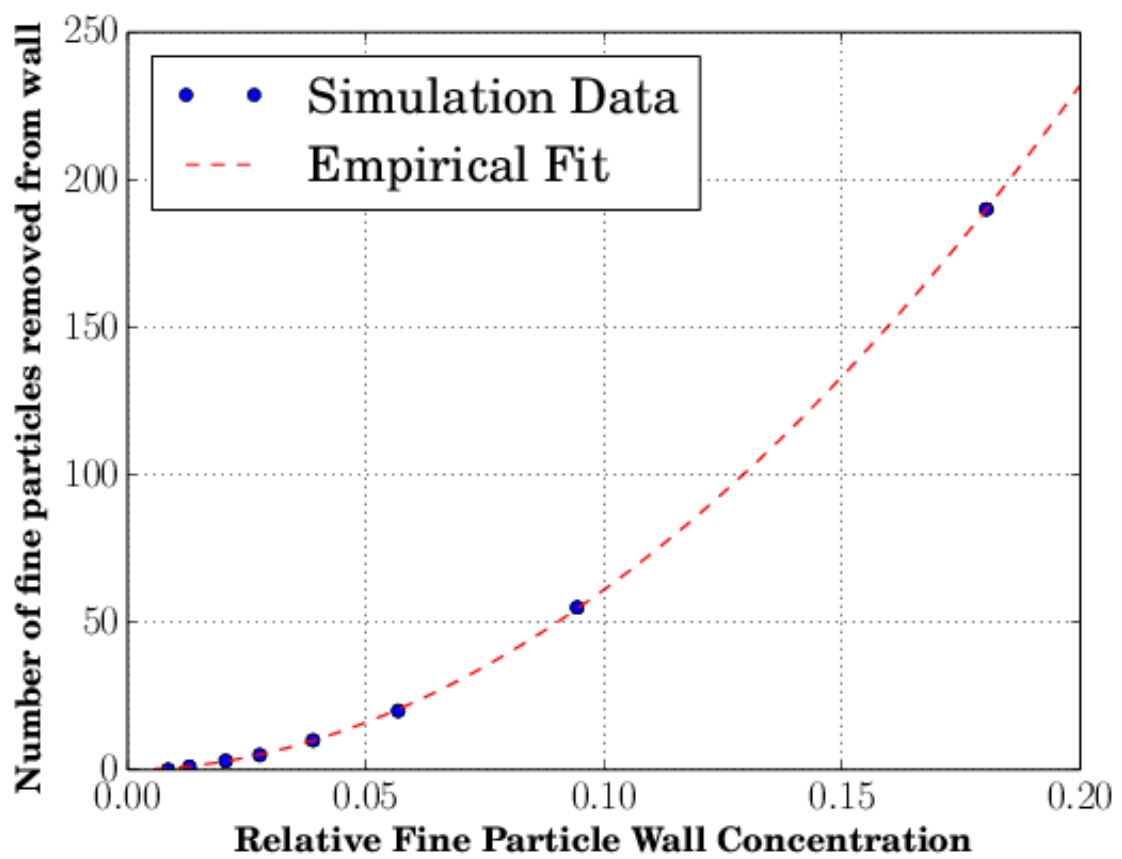

Figure 9: The simulation results (symbols) and the fit for the number of fine particles that are removed from the wall when it is hit by a carrier particle for $H a=1.0 \times 10^{-19} \mathrm{~J}$ as a function of the relative concentration of fines on the wall. 


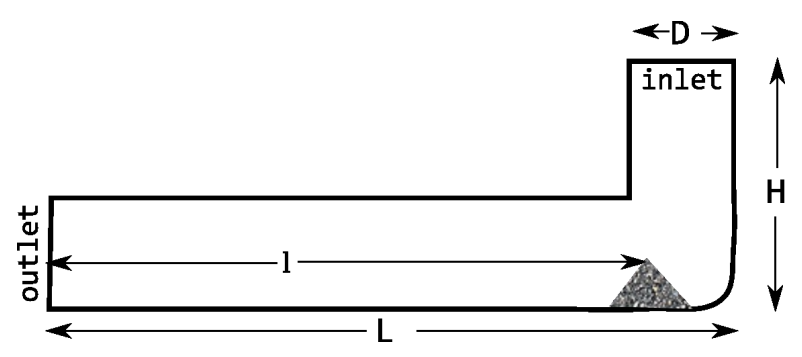

Figure 10: A sketch of the geometry of the inhaler and the initial placement of the particles. 


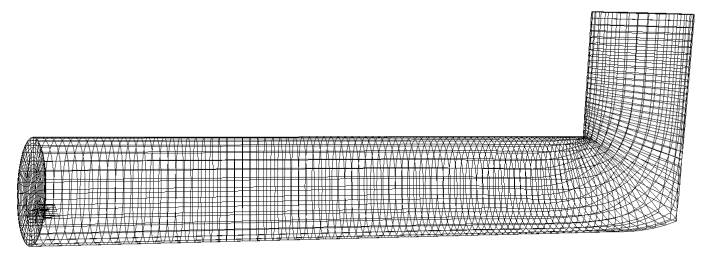

Figure 11: The geometry and the surface mesh of the inhaler geometry. 


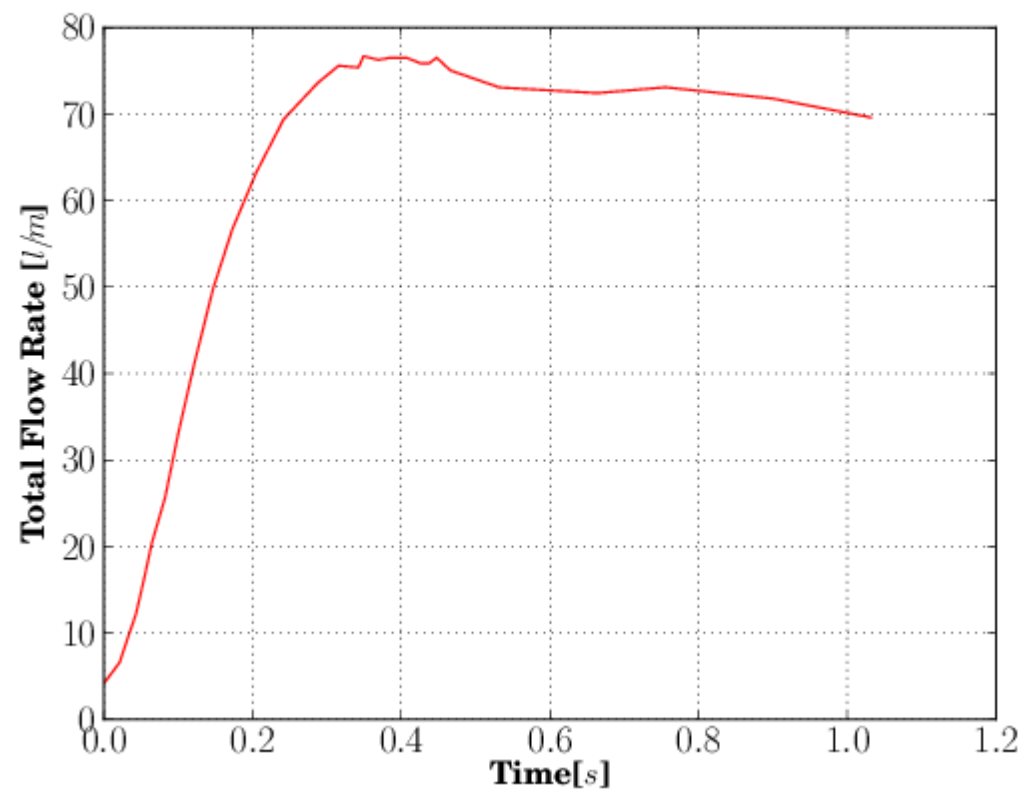

Figure 12: The flow rate, in $l / \min$, through the inhaler as a function of time. 

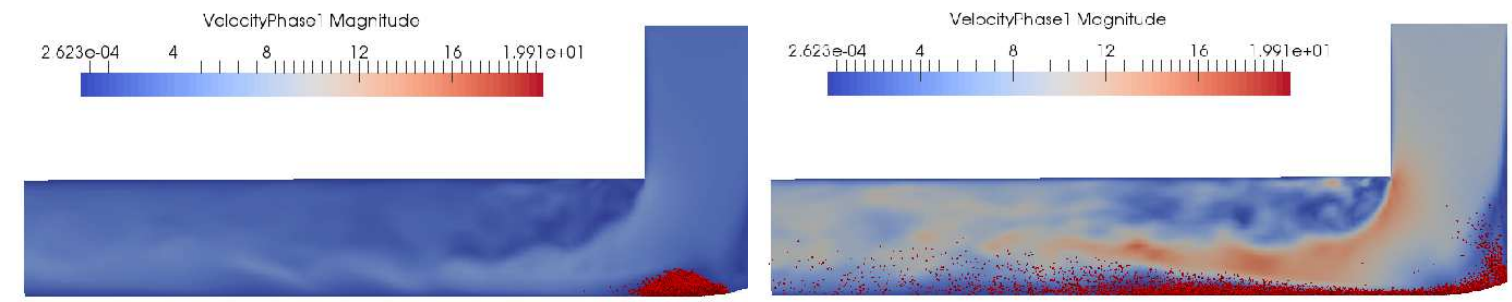

Time: $0.020000 \mathrm{~s}$

Time: $0.060000 \mathrm{~s}$

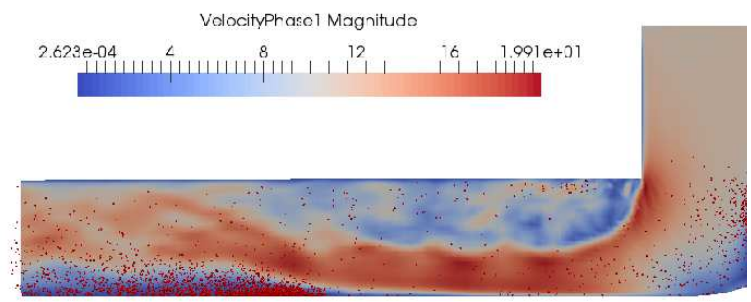

Time: $0.080000 \mathrm{~s}$

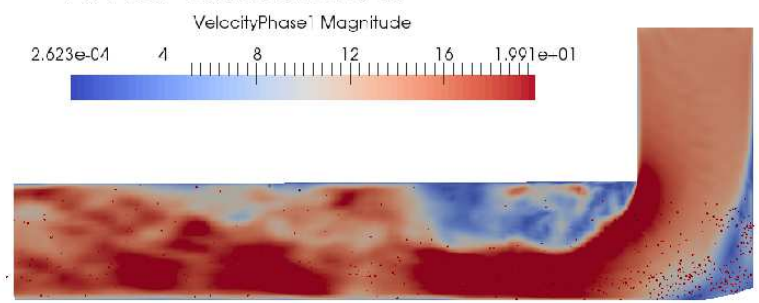

Time: $0.100000 \mathrm{~s}$

Figure 13: A sequence of two-dimensional cross sectional visualisations of the inhaler, showing the air velocity and the location of the individual carrier particles (coloured red, $d_{p}=70 \mu m$ ) for different times. 


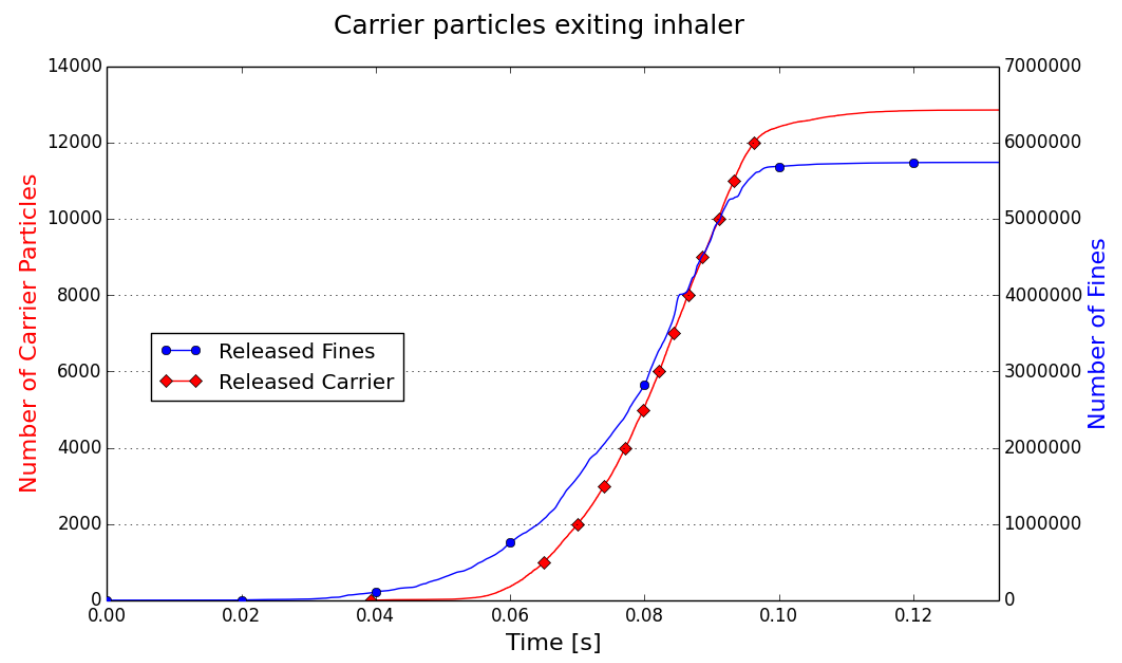

Figure 14: The number of carrier particles (red, left Y-axis) and the number of fine particles (blue, right $\mathrm{Y}$-axis) exiting the inhaler as a function of time. 


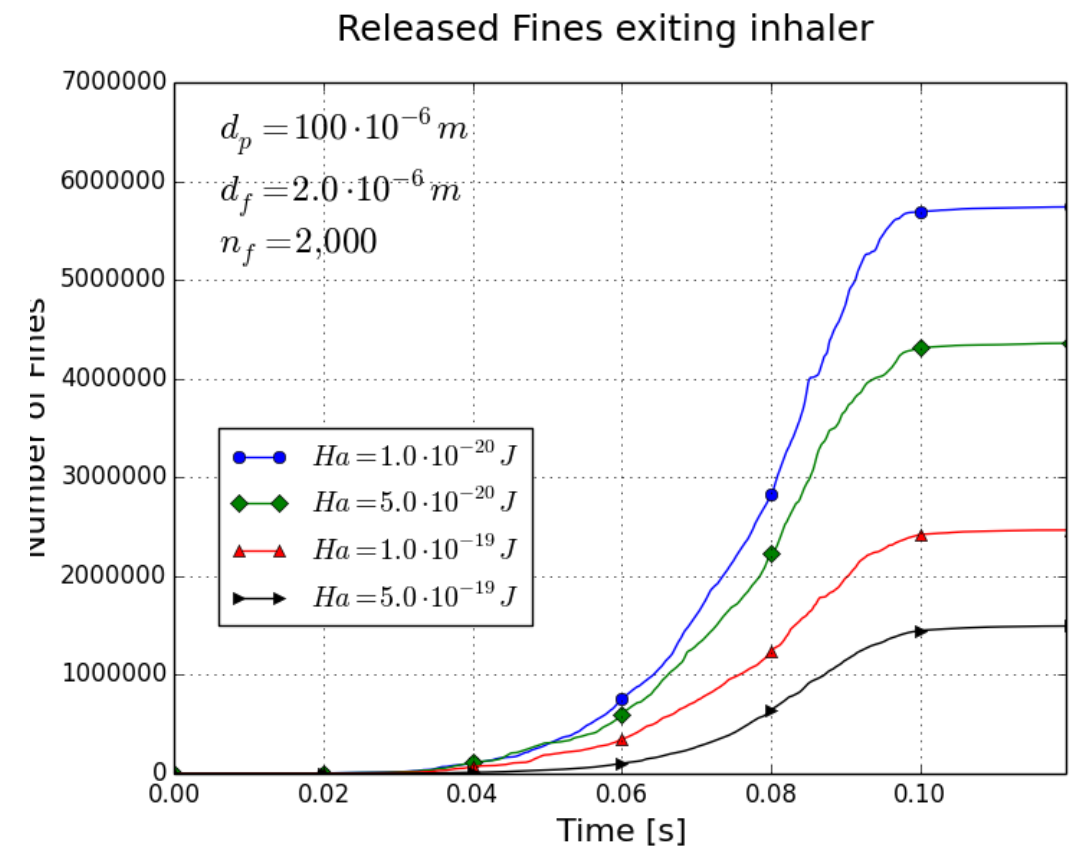

Figure 15: The cumulative number of fines that are released from the carrier particle as they exit the inhaler as a function of time, for different Hamaker values. 


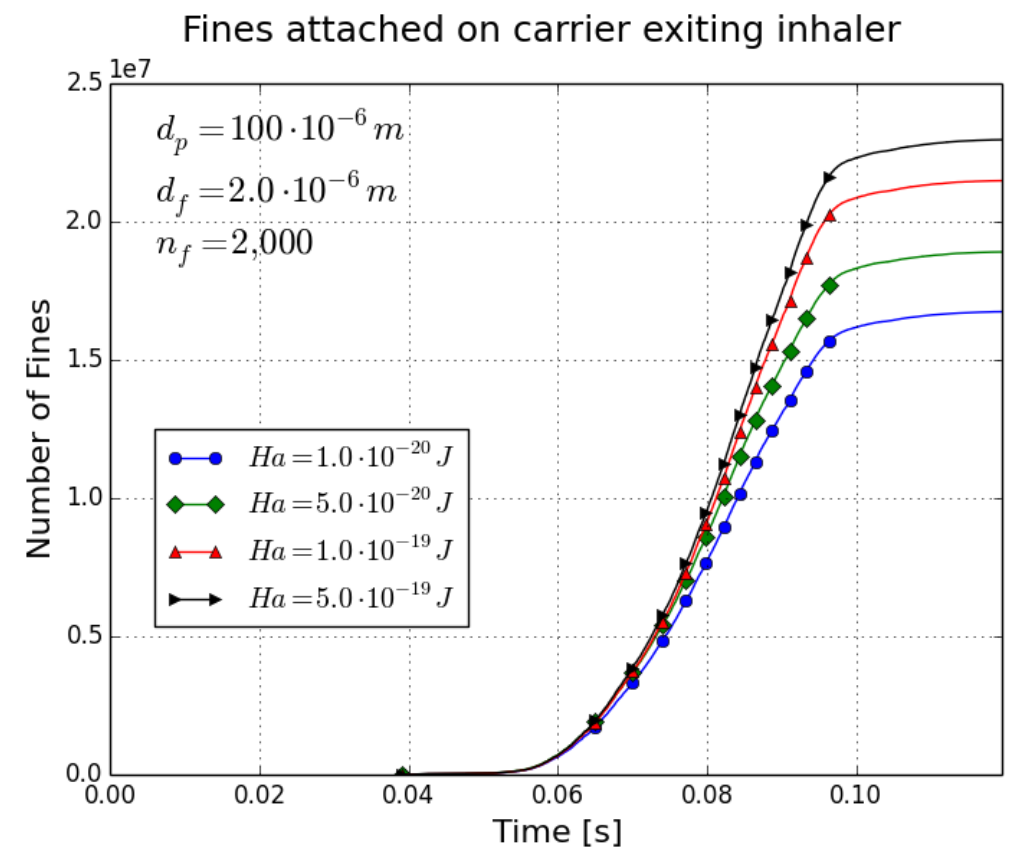

Figure 16: The cumulative number of fines that exit the inhaler, which are still attached to a carrier particle. 


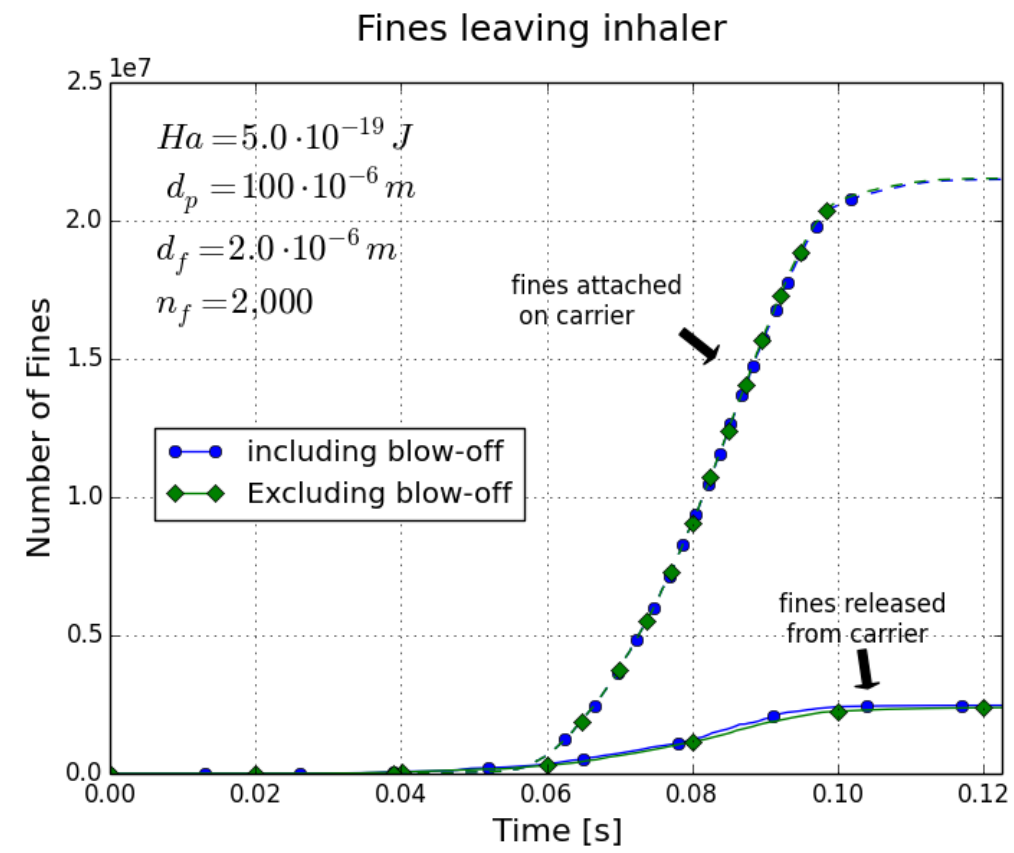

Figure 17: The cumulative number of fines that exit the inhaler, either attached to a carrier particle (dashed lines) or released (solid lines) for the case including the blow-off mechanism compared to excluding this mechanism. The results shown are for withes with Hamaker constant $H a=5.0 \cdot 10^{-19} \mathrm{~J}$. 


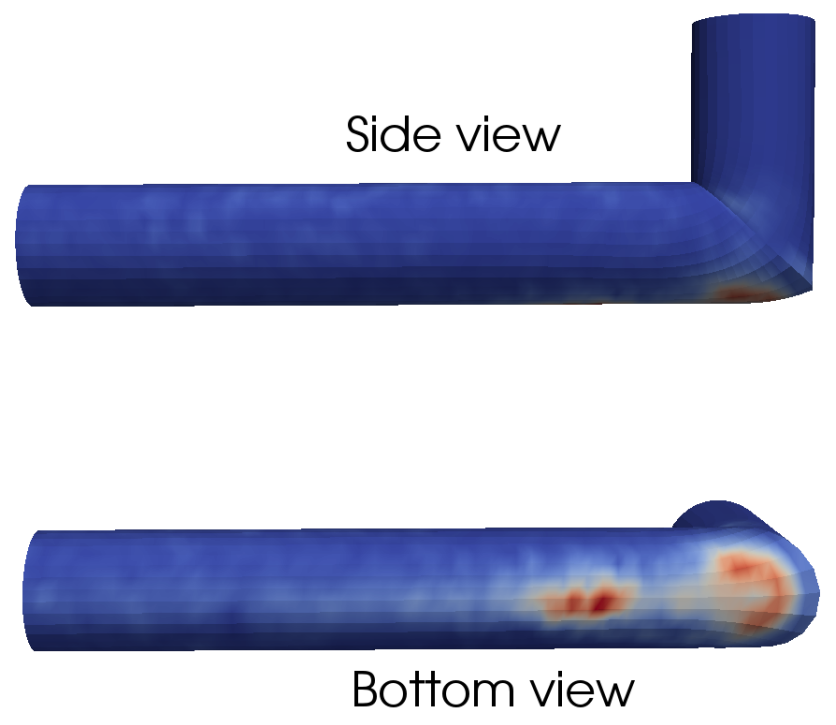

Figure 18: The location and relative concentration of the fine particles which remain adhered to the wall after all carrier particles have left the inhaler. The results shown are for the fine particles with $H a=1.0 \cdot 10^{-19} \mathrm{~J}$. 

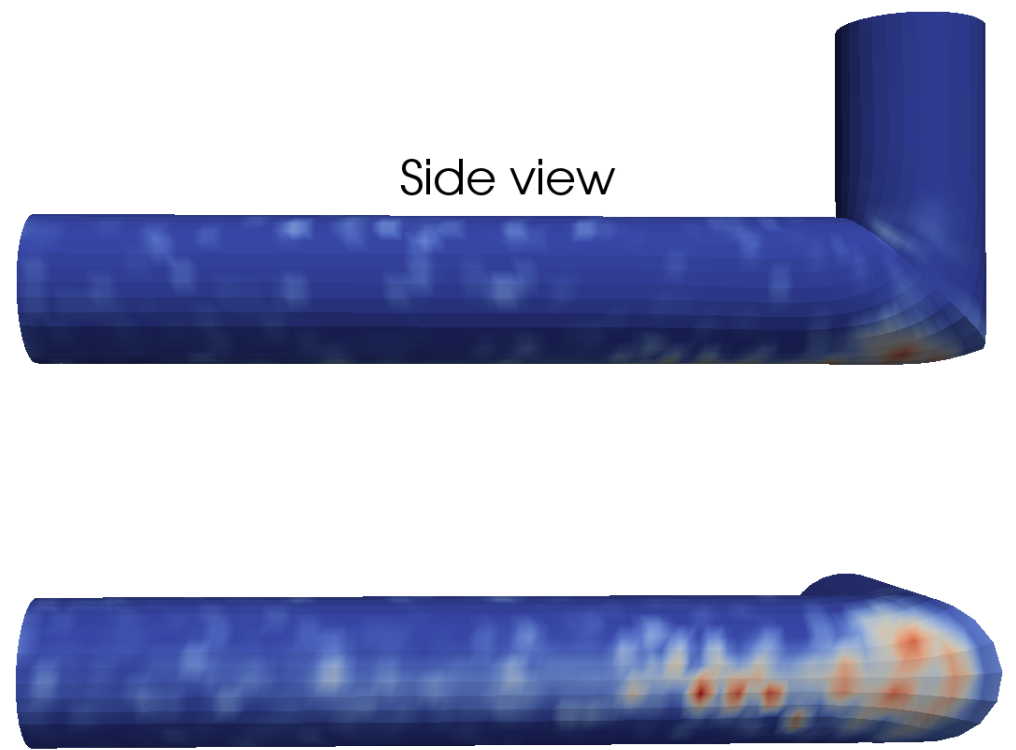

Bottom view

Figure 19: The location and relative contribution to detachment of fine particle release due to particles colliding with the wall. The results shown are for the fine particles with $H a=1.0 \cdot 10^{-19} \mathrm{~J}$. 


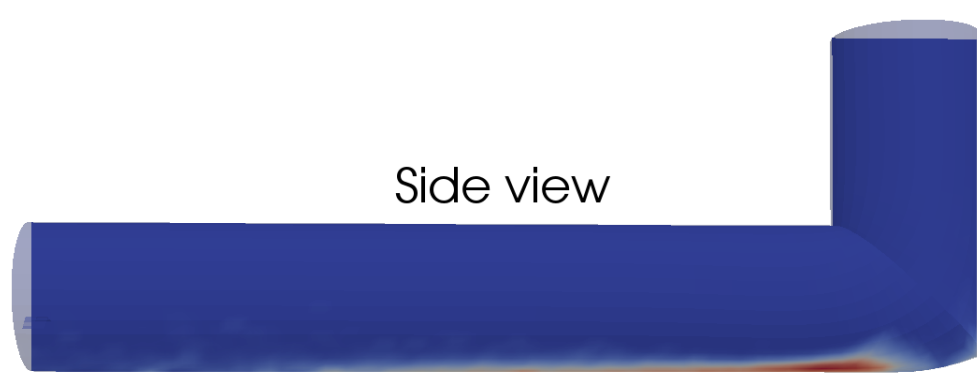

\section{Bottom view}

Figure 20: The location and relative contribution to detachment of fine particle release due to particleparticle collisions. The top image, side view, shows the cross-section through the middle of the inhaler. The results shown are for the fine particles with $\mathrm{Ha}=1.0 \cdot 10^{-19} \mathrm{~J}$. 

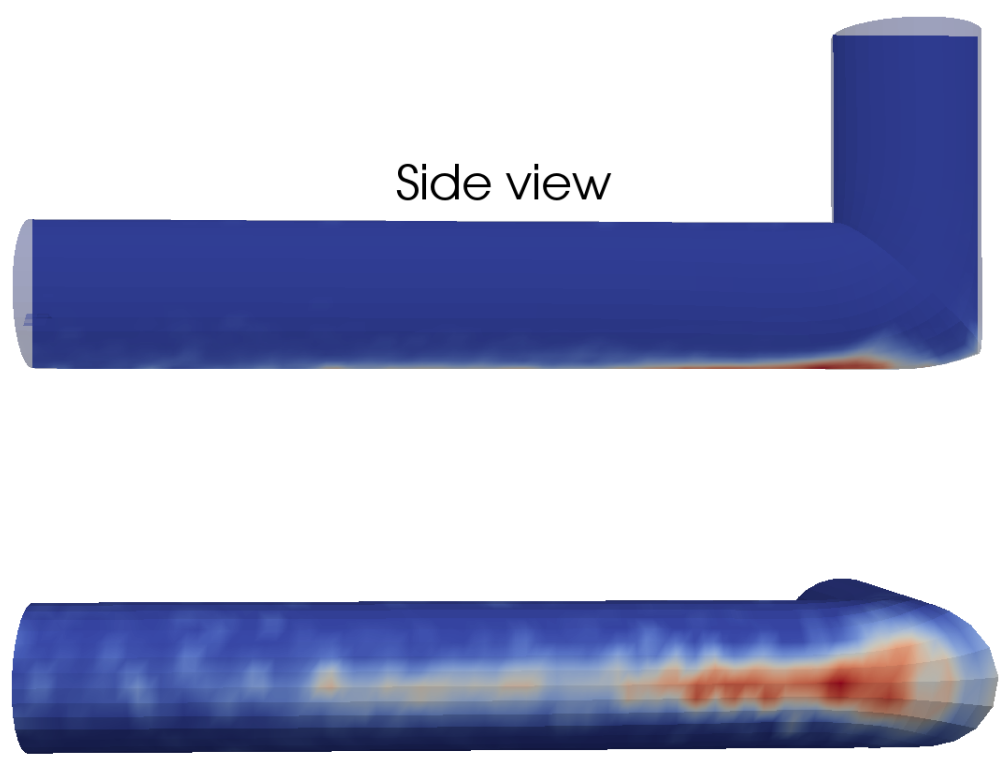

\section{Bottom view}

Figure 21: The location and relative net contribution to the detachment of fine particles for all the micro-model mechanisms considered in this paper. The top image, side view, shows the cross-section through the middle of the inhaler. The results shows are for the fine particles with $H a=1.0 \cdot 10^{-19} \mathrm{~J}$. 
Table 1: The properties of the carrier particle and the fine particles. The properties are taken from lactose reference values. ${ }^{55,56}$

\begin{tabular}{l|ll}
\hline & Carrier particle & Fine particles \\
Density $\left[\mathrm{kgm}^{-3}\right]$ & 1520 & 1520 \\
Diameter $[\mu \mathrm{m}]$ & 70 & $1.5-2.0$ \\
Youngs modulus $[\mathrm{GPa}]$ & 9.0 & 9.0 \\
Poisson's ratio [-] & 0.35 & 0.35 \\
Friction coefficient [-] & 0.3 & 0.45 \\
Restitution coefficient [-] & 0.85 & 0.65 \\
\hline
\end{tabular}


Table 2: The values of $A_{0}$ and $A_{1}$ for Equation 40, where the velocity is specified in $m s^{-1}$, as a function of Hamaker constant to predict the fraction of fines released from a coated carrier particle undergoing a collision.

\begin{tabular}{c|cc}
\hline $\mathrm{Ha}[\mathrm{J}]$ & $A_{0}$ & $A_{1}$ \\
\hline $1.0 \times 10^{-19}$ & 0.0105 & 1.042 \\
$5.0 \times 10^{-19}$ & 0.0109 & 1.473 \\
$1.0 \times 10^{-20}$ & 0.0419 & 1.150 \\
$5.0 \times 10^{-20}$ & 0.0469 & 1.228
\end{tabular}


Table 3: The values of $B_{0}$ through $B_{4}$ as a function of Hamaker constant to predict the fraction of fines released from a coated carrier particle undergoing a collision, predicted by Equation 41, where $U_{n}$ is given in $m s^{-1}$.

\begin{tabular}{c|ccccc}
\hline $\mathrm{Ha}[\mathrm{J}]$ & $B_{0}$ & $B_{1}$ & $B_{2}$ & $B_{3}$ & $B_{4}$ \\
\hline $1.0 \times 10^{-19}$ & 0.0540 & 1.109 & 4.8 & 0.158 & 0.467 \\
$5.0 \times 10^{-19}$ & 0.0492 & 1.204 & 4.6 & 0.212 & 0.403 \\
$1.0 \times 10^{-20}$ & 0.0501 & 1.245 & 4.5 & 0.261 & 0.364 \\
$5.0 \times 10^{-20}$ & 0.0473 & 1.31 & 4.4 & 0.322 & 0.318
\end{tabular}


Table 4: The values of $C_{0}$ through $C_{4}$ as a function of Hamaker constant to predict the fraction of fines released from a coated carrier particle undergoing a collision which attach directly to the wall, predicted by Equation 40

\begin{tabular}{c|ccccc}
\hline$H a$ & $C_{0}$ & $C_{1}$ & $C_{2}$ & $C_{3}$ & $C_{4}$ \\
\hline $1.0 \times 10^{-19}$ & 0.0323 & 1.243 & 4.8 & 0.286 & 0.107 \\
$5.0 \times 10^{-19}$ & 0.0283 & 1.211 & 4.6 & 0.254 & 0.0100 \\
$1.0 \times 10^{-20}$ & 0.0221 & 1.184 & 4.5 & 0.221 & 0.0921 \\
$5.0 \times 10^{-20}$ & 0.0189 & 1.154 & 4.4 & 0.194 & 0.0890
\end{tabular}


Table 5: The values of $D_{0}$ and $D_{1}$ as a function of Hamaker constant to predict the fraction of fines released from the wall, predicted by Equation 43.

\begin{tabular}{c|cc}
\hline$H a$ & $D_{0}$ & $D_{1}$ \\
\hline $1.0 \times 10^{-19}$ & 190.3 & 1.1724 \\
$5.0 \times 10^{-19}$ & 5158.8 & 1.9271 \\
$1.0 \times 10^{-20}$ & 2235.2 & 1.306 \\
$5.0 \times 10^{-20}$ & 3914.2 & 1.604
\end{tabular}


Table 6: The values of $E_{0}, E_{1}$ and $U_{0}$ as a function of Hamaker constant to predict the fraction of fines released from the carrier particle by the air flow per unit time, predicted by Equation 44, where $U$ is given in $m s^{-1}$.

\begin{tabular}{c|ccc}
\hline$H a[\mathrm{~J}]$ & $E_{0}$ & $E_{1}$ & $U_{0}\left[\mathrm{~ms}^{-1}\right]$ \\
\hline $1.0 \times 10^{-19}$ & $2.12 \cdot 10^{-6}$ & 2.89 & 13 \\
$5.0 \times 10^{-19}$ & $2.67 \cdot 10^{-5}$ & 3.26 & 10 \\
$1.0 \times 10^{-20}$ & $1.89 \cdot 10^{-4}$ & 3.01 & 8 \\
$5.0 \times 10^{-20}$ & $1.67 \cdot 10^{-3}$ & 2.82 & 5
\end{tabular}


Table 7: The percentage from the total initial number of fines which are adhered to the wall and percentage of fines which leaves the inhaler as dispersed, individual particles.

\begin{tabular}{l|llll}
$H a[\mathrm{~J}]$ & $1.0 \cdot 10^{-20}$ & $5.0 \cdot 10^{-20}$ & $1.0 \cdot 10^{-19}$ & $5.0 \cdot 10^{-19}$ \\
\hline fines attached on walls & $10.9 \%$ & $10.3 \%$ & $5.1 \%$ & $3.2 \%$ \\
fines released & $27.58 \%$ & $18.78 \%$ & $12.75 \%$ & $8.56 \%$
\end{tabular}

